\title{
Existence of Entropy Solutions to Two-Dimensional Steady Exothermically Reacting Euler Equations
}

\author{
Gui-Qiang Chen, Changguo Xiao \& Yongqian Zhang \\ Mathematical Institute, University of Oxford, Oxford, OX2 6GG, UK; \\ School of Mathematical Sciences, Fudan University, Shanghai 200433, China. \\ E-mail: chengq@maths.ox.ac.uk \\ School of Mathematical Sciences, Fudan University, Shanghai 200433, China. \\ E-mail:09110180021@fudan.edu.cn; yongqianz@fudan.edu.cn
}

\begin{abstract}
We are concerned with the global existence of entropy solutions of the two-dimensional steady Euler equations for an ideal gas, which undergoes a one-step exothermic chemical reaction under the Arrhenius-type kinetics. The reaction rate function $\phi(T)$ is assumed to have a positive lower bound. We first consider the Cauchy problem (the initial value problem), that is, seek a supersonic downstream reacting flow when the incoming flow is supersonic, and establish the global existence of entropy solutions when the total variation of the initial data is sufficiently small. Then we analyze the problem of steady supersonic, exothermically reacting Euler flow past a Lipschitz wedge, generating an additional detonation wave attached to the wedge vertex, which can be then formulated as an initial-boundary value problem. We establish the globally existence of entropy solutions containing the additional detonation wave (weak or strong, determined by the wedge angle at the wedge vertex) when the total variation of both the slope of the wedge boundary and the incoming flow is suitably small. The downstream asymptotic behavior of the global solutions is also obtained.
\end{abstract}

2010 Mathematics Subject Classification: 35L65; 76N10; 35B40; 35A01; 35L45; 35L50; 35L67; $76 \mathrm{~V} 05$

Keywords: Combustion, detonation wave, stability, Glimm scheme, fractional-step, supersonic flow, reacting Euler flow, Riemann problem, entropy solutions, two-dimensional, steady flow, asymptotic behavior.

\section{Introduction}

We are concerned with the two-dimensional steady supersonic Euler flow of an exothermically reacting ideal gas, which is governed by

$$
\begin{array}{r}
(\rho u)_{x}+(\rho v)_{y}=0, \\
\left(\rho u^{2}+p\right)_{x}+(\rho u v)_{y}=0, \\
(\rho u v)_{x}+\left(\rho v^{2}+p\right)_{y}=0, \\
((\rho E+p) u)_{x}+((\rho E+p) v)_{y}=0, \\
(\rho u Z)_{x}+(\rho v Z)_{y}=-\rho Z \phi(T) .
\end{array}
$$

Here $(u, v)$ is the velocity, $p$ the scalar pressure, $\rho$ the density, $Z$ the fraction of unburned gas in the mixture, $\phi(T)$ the reaction rate, $q$ the specific binding energy of unburned gas, and 
$E=\frac{1}{2}\left(u^{2}+v^{2}\right)+e(\rho, p)+q Z$ the specific total energy with the specific internal energy $e$ that is a given function of $(\rho, p)$ defined through thermodynamical relations.

For an ideal gas,

$$
p=R \rho T, \quad e=c_{v} T, \quad \gamma=1+\frac{R}{c_{v}}>1,
$$

where $R$ and $c_{v}$ are positive constants, and $\gamma$ is the adiabatic exponent. We identify $c_{v}+R=c_{p}$ as the specific heat at constant pressure.

We assume for simplicity that the specific heats and molecular weights of the reactant and product gases are the same and that the reaction rate function $\phi$ is monotonically increasing and Lipschitz continuous. In addition, inadmissible discontinuous solutions are eliminated by requiring the following entropy condition:

$$
(\rho u S)_{x}+(\rho v S)_{y} \geq \frac{q \rho Z \phi(T)}{T} .
$$

We first consider the Cauchy problem (the initial value problem) for (1.1) -(1.5) in the region $\{x \geq 0, y \in \mathbb{R}\}$, with initial incoming flow (initial data):

$$
(u, v, p, \rho, Z)(0, y)=\left(u_{0}, v_{0}, p_{0}, \rho_{0}, Z_{0}\right)(y), \quad y \in \mathbb{R} .
$$

We assume that $u_{0}(y), v_{0}(y), p_{0}(y), \rho_{0}(y)$, and $Z_{0}(y)$ are bounded and have bounded total variation with $Z_{0}(-\infty)=\lim _{y \rightarrow-\infty} Z_{0}(y)=0$. We further assume that there are positive constants $u^{\prime}, \rho^{\prime}$, and $T^{\prime}$ such that

$$
u_{0}>c_{0} \geq u^{\prime}>0, \quad \rho_{0} \geq \rho^{\prime}>0, \quad T_{0} \geq T^{\prime}>0,
$$

where $c=\sqrt{\frac{\gamma p}{\rho}}$ is the local sonic speed. We make this assumption on the initial data to ensure that the flow is supersonic (i.e. $\left.u^{2}+v^{2}>c^{2}\right)$.

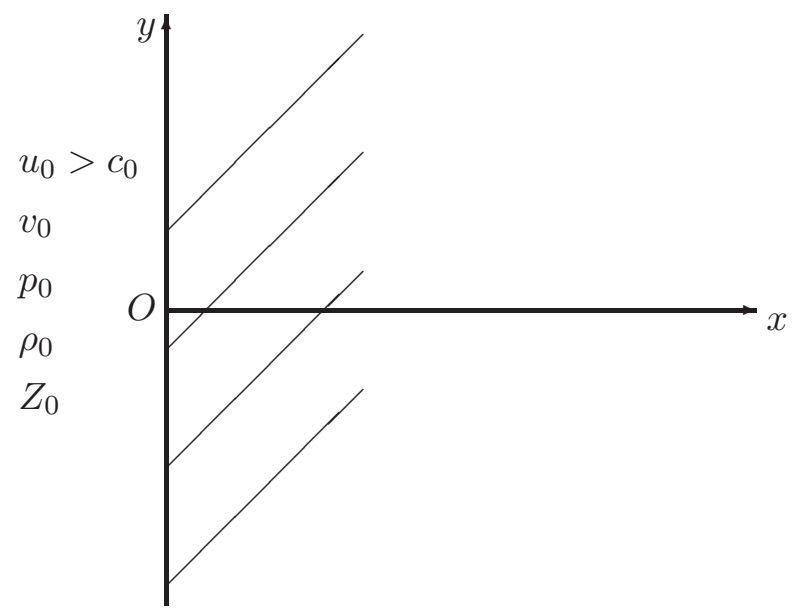

Fig.1. Supersonic Euler flow through the left boundary $x=0$

We assume the initial data to be such that the reaction rate function $\phi(T)$ never vanishes, so that there is a positive minimum value $\Phi:=\phi\left(T^{\prime}\right)>0$. In a sense, this is a very realistic condition. Typically, $\phi(T)$ has the Arrhenius form:

$$
\phi(T)=T^{\alpha} e^{-\frac{E}{R T}},
$$

which vanishes only at absolutely zero temperature, where $\alpha$ is a positive constant. We make this assumption in order to obtain the uniform decay of the reactant to zero. Although the total 
variation of the solution may very well increase while the reaction is active, the reaction must eventually die out along the flow trajectories. Consequently, the increase in total variation can be estimated rigorously.

In Chen-Wagner [1], the large-time existence of entropy solutions to the Cauchy problem has been established for the time-dependent equations of planar flow of an exothermically reacting ideal gas. The total variation of the initial data is bounded by a parameter $\epsilon=\gamma-1$, which grows arbitrarily large as $\epsilon \rightarrow 0$ whose limiting case is the isothermal gas. Global entropy solutions are obtained by using the Glimm fractional-step scheme based on the Glimm scheme.

In this paper, we first establish a global existence theory for entropy solutions of the Cauchy problem for two-dimensional, exothermically reacting steady Euler equations by further developing the Glimm scheme, under the condition that the total variation of the initial data in (1.8) is small. Then this approach is further developed for solving the supersonic reacting Euler flow past Lipschitz wedges. For a non-reacting supersonic flow past a straight wedge, an attached plane shock is generated at the wedge vertex. When the supersonic flow is governed by the exothermically reacting steady Euler equations, the attached detonation wave is no longer a plane wave even for the straight wedge, whose strength (weak or strong) is determined by the wedge angle and the incoming flow. Nevertheless, we establish that, when the total variation of both the incoming supersonic flow and the slope of the wedge boundary is suitably small, there exists a global entropy solution containing the (weak or strong) detonation wave. The downstream asymptotic behavior of entropy solutions is also obtained.

The organization of this paper is as follows. In Section 2, we discuss some basic features of the exothermically reacting Euler equations (1.1)-(1.5). The Glimm fractional-step scheme is described for the Cauchy problem (1.8) for system (1.1)-(1.5) in Section 3. In Section 4, we establish uniform bounds on the total variation in the $y$-direction of the Glimm fractional-step approximate solutions for the Cauchy problem (1.8). In Section 5, we establish uniform bounds on the total variation of the Glimm fractional-step approximate solutions in the $y$-variable for the initial-boundary value problem (5.1) - (5.2) for (1.1)-(1.5) concerning the supersonic reacting Euler flow past Lipschitz wedges, when the wedge angle at the wedge vertex is small. In Section 6 , the convergence of approximate solutions to an entropy solution is established for both the Cauchy problem (1.8) and the initial-boundary value problem (5.1)-(5.2) for (1.1)-(1.5). The downstream asymptotic behavior of entropy solutions is also clarified in Section 7. In Section 8, we extend the results in Sections 5-6 for the case of small wedge angle to the case of large wedge angle, for which the entropy solution contains a strong detonation wave generated between the incoming fluid and the wedge boundary at the wedge vertex.

\section{Basic Features of the Exothermically Reacting Euler Equations}

In this section, we discuss some basic features of system (1.1)-(1.5).

\subsection{Euler equations}

System (1.1) - (1.5) can be rewritten in the following form:

$$
W(U)_{x}+H(U)_{y}=G(U),
$$

with $U=(u, v, p, \rho, Z)$, where

$$
\begin{aligned}
& W(U)=\left(\rho u, \rho u^{2}+p, \rho u v, \rho u\left(\bar{h}+\frac{u^{2}+v^{2}}{2}\right), \rho u Z\right), \\
& H(U)=\left(\rho v, \rho u v, \rho v^{2}+p, \rho v\left(\bar{h}+\frac{u^{2}+v^{2}}{2}\right), \rho v Z\right), \\
& G(U)=(0,0,0, q \rho \phi(T) Z,-\rho \phi(T) Z)
\end{aligned}
$$


with $\bar{h}=\frac{\gamma p}{(\gamma-1) \rho}$.

In the case when $G(U)$ is identically zero, system (2.1) becomes a system of conservation laws:

$$
W(U)_{x}+H(U)_{y}=0 .
$$

For a smooth solution $U(x, y)$, system (2.2) is equivalent to

$$
\nabla_{U} W(U) U_{x}+\nabla_{U} H(U) U_{y}=0 .
$$

Then the eigenvalues of (2.2) are the roots of the 5th order polynomial

$$
\operatorname{det}\left(\lambda \nabla_{U} W(U)-\nabla_{U} H(U)\right)
$$

that is, the solutions of the equation:

$$
(v-\lambda u)^{3}\left((v-\lambda u)^{2}-c^{2}\left(1+\lambda^{2}\right)\right)=0,
$$

where $c=\sqrt{\frac{\gamma p}{\rho}}$ is the sonic speed.

If the flow is supersonic (i.e. $u^{2}+v^{2}>c^{2}$ ), system (2.1) is hyperbolic. In particular, when $u>c$, the system has five eigenvalues in the $x$-direction:

$$
\lambda_{i}=\frac{v}{u}, \quad i=2,3,4 ; \quad \lambda_{j}=\frac{u v+(-1)^{\frac{j+3}{4}} c \sqrt{u^{2}+v^{2}-c^{2}}}{u^{2}-c^{2}}, \quad j=1,5,
$$

and the corresponding linearly independent eigenvectors:

$$
\begin{gathered}
r_{j}=\kappa_{j}\left(-\lambda_{j}, 1, \rho\left(\lambda_{j} u-v\right), \frac{\rho\left(\lambda_{j} u-v\right)}{c^{2}}, 0\right)^{\top}, \quad j=1,5 ; \\
r_{2}=(u, v, 0,0,0)^{\top}, \quad r_{3}=(0,0,0, \rho, 0)^{\top}, \quad r_{4}=\left(0,0,0,0, \frac{1}{\rho u}\right)^{\top},
\end{gathered}
$$

where $\kappa_{j}$ are chosen so that $r_{j} \cdot \nabla \lambda_{j}=1$ since the $j$ th-characteristic fields are genuinely nonlinear, $j=1,5$. Note that $r_{j} \cdot \nabla \lambda_{j}=0, j=2,3,4$, that is, these characteristic fields are always linearly degenerate.

In particular, at a constant state $\tilde{U}=(\tilde{u}, 0, \tilde{p}, \tilde{\rho}, \tilde{Z})$,

$$
\lambda_{2}(\tilde{U})=\lambda_{3}(\tilde{U})=\lambda_{4}(\tilde{U})=0, \quad \lambda_{1}(\tilde{U})=-\frac{\tilde{c}}{\sqrt{\tilde{u}^{2}-\tilde{c}^{2}}}=-\lambda_{5}(\tilde{U})<0 .
$$

Definition 2.1 (Entropy Solutions). A function $U=U(x, y) \in B V\left(\mathbb{R}^{+} \times \mathbb{R}\right)$ is called an entropy solution of problem (1.8) for system (1.1) -(1.5) provided that

(i) $U$ is a weak solution of problem (1.8) for system (1.1)-(1.5), that is,

$$
\int_{-\infty}^{\infty} \int_{0}^{\infty}\left(W(U) \phi_{x}+H(U) \phi_{y}+G(U) \phi\right) d x d y+\int_{-\infty}^{\infty} W\left(U_{0}(y)\right) \phi(0, y) d y=0
$$

for any $\phi \in C_{0}^{\infty}([0, \infty) \times(-\infty, \infty))$;

(ii) For any convex entropy pair $(\eta, q)$ with respect to $W(U)$, the following inequality

$$
\eta(W(U))_{x}+q(W(U))_{y} \leq \nabla_{W} \eta(W(U)) G(U)
$$

holds in the sense of distributions, that is,

$$
\begin{array}{r}
\int_{-\infty}^{\infty} \int_{0}^{\infty}\left(\eta(W(U)) \phi_{x}+q(W(U)) \phi_{y}+\nabla_{W} \eta(W(U)) G(U) \phi\right) d x d y \\
+\int_{-\infty}^{\infty} \eta\left(W\left(U_{0}(y)\right)\right) \phi(0, y) d y \geq 0
\end{array}
$$

for any $\phi \in C_{0}^{\infty}([0, \infty) \times(-\infty, \infty))$ and $\phi(x, y) \geq 0$. 
Remark 2.1. In particular, $\eta(W)=-\rho u S$ is an entropy which is convex with respect to $W$, while $q(W)=-\rho v S$ is the corresponding entropy flux, when $u>c>0$.

As in [1], if we rewrite system (2.2) in Lagrangian coordinates:

$$
\left(x^{\prime}, m\right)=(x, m(x, y))
$$

with $\mathrm{d} m=\rho u \mathrm{~d} y-\rho v \mathrm{~d} x$, then the fifth equation in (2.2) becomes

$$
Z_{x^{\prime}}=0 .
$$

It states that the $Z$-component is decoupled from $(u, v, p, \rho)^{\top}$ in the solution of the non-reacting Riemann problem.

\subsection{Wave curves in the phase space}

We now analyze some basic properties of nonlinear waves. We focus on the case when $u>c>0$ in the state space. Seek the self-similar solutions to system (2.2):

$$
(u, v, p, \rho, Z)(x, y)=(u, v, p, \rho, Z)(\xi), \quad \xi=\frac{y}{x},
$$

which connect to a fixed constant state $U_{0}=\left(u_{0}, v_{0}, p_{0}, \rho_{0}, z_{0}\right)$. Then we have

$$
\operatorname{det}\left(\xi \nabla_{U} W(U)-\nabla_{U} H(U)\right)=0,
$$

which implies

$$
\xi=\lambda_{i}(U)=\frac{v}{u}, \quad i=2,3,4 ; \quad \text { or } \quad \xi=\lambda_{j}(U), \quad j=1,5 .
$$

Plugging $\xi=\lambda_{i}(U), i=2,3,4$, into (2.2), we obtain

$$
d p=0, \quad v d u-u d v=0,
$$

which yields the contact discontinuity curves $C_{i}\left(U_{0}\right)$ in the phase space:

$$
C_{i}\left(U_{0}\right): p=p_{0}, w=\frac{v}{u}=\frac{v_{0}}{u_{0}}, \quad i=2,3,4 .
$$

More precisely, we have

$$
C_{2}\left(U_{0}\right): U=\left(u_{0} e^{\sigma_{2}}, v_{0} e^{\sigma_{2}}, p_{0}, \rho_{0}, Z_{0}\right)^{\top},
$$

with strength $\sigma_{2}$ and slope $\frac{v_{0}}{u_{0}}$, which is determined by

$$
\left\{\begin{array}{l}
\frac{d U}{d \sigma_{2}}=r_{2}(U), \\
\left.U\right|_{\sigma_{2}=0}=U_{0}
\end{array}\right.
$$

and

$$
C_{3}\left(U_{0}\right): U=\left(u_{0}, v_{0}, p_{0}, \rho_{0} e^{\sigma_{3}}, Z_{0}\right)^{\top},
$$

with strength $\sigma_{3}$ and slope $\frac{v_{0}}{u_{0}}$, which is determined by

$$
\left\{\begin{array}{l}
\frac{d U}{d \sigma_{3}}=r_{3}(U), \\
\left.U\right|_{\sigma_{3}=0}=U_{0}
\end{array}\right.
$$

and

$$
C_{4}\left(U_{0}\right): U=\left(u_{0}, v_{0}, p_{0}, \rho_{0}, Z_{0}+\frac{\sigma_{4}}{\rho_{0} u_{0}}\right)^{\top}
$$


with strength $\sigma_{4}$ and slope $\frac{v_{0}}{u_{0}}$, which is determined by

$$
\left\{\begin{array}{l}
\frac{d U}{d \sigma_{4}}=r_{4}(U) \\
\left.U\right|_{\sigma_{4}}=0=U_{0}
\end{array}\right.
$$

We can see that $\sigma_{4}$ is the difference between $w_{5}=\rho u Z$ in the Riemann problem.

Plugging $\xi=\lambda_{j}(U), j=1,5$, into (2.2), we obtain the $j$-th rarefaction wave curve $R_{j}\left(U_{0}\right)$, $j=1,5$, in the phase space through $U_{0}$ :

$$
R_{j}\left(U_{0}\right): d p=c^{2} d \rho, d u=-\lambda_{j} d v, \rho\left(\lambda_{j} u-v\right) d v=d p, d Z=0, \quad j=1,5 .
$$

Now we consider discontinuous solutions so that the equations in (2.2) are satisfied in the distributional sense. This implies that the following Rankine-Hugoniot conditions hold along the discontinuity with speed $s$, which connects to a state $U_{0}=\left(u_{0}, v_{0}, p_{0}, \rho_{0}, Z_{0}\right)$ :

$$
\begin{aligned}
s[\rho u] & =[\rho v], \\
s\left[\rho u^{2}+p\right] & =[\rho u v], \\
s[\rho u v] & =\left[\rho v^{2}+p\right], \\
s\left[\rho u\left(\bar{h}+\frac{u^{2}+v^{2}}{2}\right)\right] & =\left[\rho v\left(\bar{h}+\frac{u^{2}+v^{2}}{2}\right)\right], \\
s[\rho u Z] & =[\rho v Z],
\end{aligned}
$$

where the jump symbol [.] stands for the value of the quantity of the front-state minus that of the back-state. Then we have

$$
\left(v_{0}-s u_{0}\right)^{3}\left(\left(v_{0}-s u_{0}\right)^{2}-\bar{c}^{2}\left(1+s^{2}\right)\right)=0,
$$

where $\bar{c}^{2}=\frac{c_{0}^{2}}{b} \frac{\rho}{\rho_{0}}$ and $b=\frac{\gamma+1}{2}-\frac{\gamma-1}{2} \frac{\rho}{\rho_{0}}$. This implies

$$
s=s_{i}=\frac{v_{0}}{u_{0}}, \quad i=2,3,4,
$$

or

$$
s=s_{j}=\frac{u_{0} v_{0}+(-1)^{\frac{j+3}{4}} \bar{c} \sqrt{u_{0}^{2}+v_{0}^{2}-\bar{c}^{2}}}{u_{0}^{2}-\bar{c}^{2}}, \quad j=1,5,
$$

where $u_{0}>\bar{c}$ for small shocks.

Plugging $s_{i}, i=2,3,4$, into (2.24)-(2.28), we obtain the same $C_{i}\left(U_{0}\right), i=2,3,4$, as defined in (2.17), (2.19), and (2.21); while plugging $s_{j}, j=1,5$, into (2.24)-(2.28), we obtain the $j$ th shock wave curve $S_{j}\left(U_{0}\right), j=1,5$, through $U_{0}$ :

$$
S_{j}\left(U_{0}\right):[p]=\frac{c_{0}^{2}}{b}[\rho],[u]=-s_{j}[v], \rho_{0}\left(s_{j} u_{0}-v_{0}\right)[v]=[p], \quad[Z]=0 .
$$

Note that the shock wave curve $S_{j}\left(U_{0}\right)$ contacts with $R_{j}\left(U_{0}\right)$ at $U_{0}$ up to second order.

Following Lax [9], we can parameterize any physically admissible wave curve in a neighborhood of a constant $\tilde{U}, O_{\epsilon}(\tilde{U})$, by $\sigma_{j} \mapsto \Phi_{j}\left(\sigma_{j}, U_{b}\right)$, with $\Phi_{j} \in C^{2},\left.\Phi_{j}\right|_{\sigma_{j}=0}=U_{b}$, and $\left.\frac{\partial \Phi_{j}}{\partial \sigma_{j}}\right|_{\sigma_{j}=0}=r_{j}\left(U_{b}\right)$. Set

$$
\Phi\left(\sigma_{5}, \sigma_{4}, \sigma_{3}, \sigma_{2}, \sigma_{1} ; U_{b}\right)=\Phi_{5}\left(\sigma_{5}, \Phi_{4}\left(\sigma_{4}, \Phi_{3}\left(\sigma_{3}, \Phi_{2}\left(\sigma_{2}, \Phi_{1}\left(\sigma_{1} ; U_{b}\right)\right)\right)\right)\right) .
$$


We denote $\Psi_{j}\left(\sigma_{j}, W\left(U_{b}\right)\right)=W\left(\Phi_{j}\left(\sigma_{j} ; U_{b}\right)\right)$ and

$$
\begin{aligned}
\Psi\left(\sigma_{5}, \sigma_{4}, \sigma_{3}, \sigma_{2}, \sigma_{1} ; W\left(U_{b}\right)\right) & =\Psi_{5}\left(\sigma_{5}, \Psi_{4}\left(\sigma_{4}, \Psi_{3}\left(\sigma_{3}, \Psi_{2}\left(\sigma_{2}, \Psi_{1}\left(\sigma_{1} ; W\left(U_{b}\right)\right)\right)\right)\right)\right) \\
& =W\left(\Phi\left(\sigma_{5}, \sigma_{4}, \sigma_{3}, \sigma_{2}, \sigma_{1} ; U_{b}\right)\right)
\end{aligned}
$$

Finally, we denote

$$
\boldsymbol{\sigma}=\left(\sigma_{1}, \sigma_{2}, \sigma_{3}, \sigma_{4}, \sigma_{5}\right)
$$

and

$$
\Psi\left(\sigma, W\left(U_{b}\right)\right)=\Psi_{5}\left(\sigma_{5}, \Psi_{4}\left(\sigma_{4}, \Psi_{3}\left(\sigma_{3}, \Psi_{2}\left(\sigma_{2}, \Psi_{1}\left(\sigma_{1}, W\left(U_{b}\right)\right)\right)\right)\right)\right) .
$$

\section{The Glimm Fractional-Step Scheme}

We employ a fractional-step scheme for the inhomogeneous system (2.1) as described in [1] based on the Glimm scheme. As before, we regard the $x$-direction as the time-like direction.

Choose mesh lengths $h>0$ and $l>0$ in the $x$-direction and $y$-direction, respectively, such that the Courant-Friedrichs-Levy condition holds:

$$
\Lambda=\max _{1 \leq j \leq 5}\left|\lambda_{j}(U)\right| \leq \frac{l}{2 h} .
$$

Partition $\mathbb{R}^{+}$by the sequence $x_{k}=k h, k \in \mathbb{Z}^{+}$, and partition $\mathbb{R}$ into cells with the $j$ th cell centered at

$$
y_{j}=j l, \quad j=0, \pm 1, \pm 2, \cdots .
$$

We begin with approximating the initial data $U_{0}(y)$ by a function $U^{h}(0, y)$, which is constant for $y$ in the interval $\left[y_{j-1}, y_{j+1}\right]$ for $j$ even and converges to $U_{0}(y)$ both pointwise a.e. and in $L^{1}$ on any bounded interval as $h \rightarrow 0$. Choose a random sequence $\theta_{k}, k=0,1,2, \cdots$, in the interval $(-1,1)$ with the uniform probability distribution.

We then construct the approximate solution $W\left(U^{h}(x, y)\right)$ as follows:

Assume that $W\left(U^{h}(x, y)\right)$ is defined for $x<k h$. Then we construct the approximate solution $W\left(U^{h}(x, y)\right)$ in the strip $[k h,(k+1) h) \times(-\infty, \infty)$ as follows:

Step 1 (Random step): Define

$$
\begin{aligned}
& W\left(U_{j}^{k}\right)=W\left(U^{h}\left(k h-,\left(j+\theta_{k}\right) l\right)\right), \\
& W\left(U^{h}(k h+0, y)\right) \equiv W\left(U_{j}^{k}\right), \quad(j-1) l \leq y<(j+1) l,
\end{aligned}
$$

where $j+k$ is even, and $\chi_{k}$ is the $k$ th element of the random sequence $\left(\chi_{1}, \cdots, \chi_{k}, \cdots\right)$.

Step 2 (Solving the Riemann problem): In the strip $[k h,(k+1) h) \times(-\infty, \infty)$, we solve the following Riemann problem in each domain $(k h,(k+1) h) \times((j-1) l,(j+1) l)$ :

$$
\left\{\begin{array}{l}
W(U)_{\tau}+H(U)_{y}=0, \\
\left.W(U)\right|_{\tau=0}= \begin{cases}W\left(U_{j-1}^{k}\right) & y<j l, \\
W\left(U_{j+1}^{k}\right) & y>j l,\end{cases}
\end{array}\right.
$$

where $j+k$ is odd and $\tau=x-k h$. The resulting solution is denoted as $W\left(U_{0}^{h}(x, y)\right)$. 


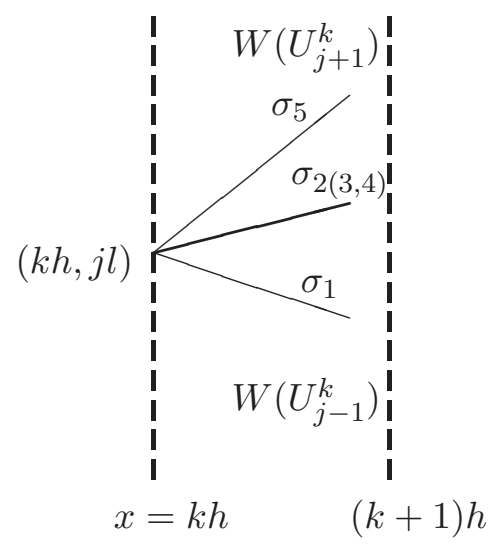

Fig. 2. Riemann problem

Step 3 (Reacting step): Define

$$
W\left(U^{h}(x, y)\right)=W\left(U_{0}^{h}(x, y)\right)+G\left(U_{0}^{h}(x, y)\right)(x-k h),
$$

where $G(U)=(0,0,0, q \rho Z \phi(T),-\rho Z \phi(T))$ as before and $k h \leq x<(k+1) h$.

Therefore, we can construct the approximate solution $W\left(U^{h}(x, y)\right)$ in the strip $[k h,(k+$ 1)h) $\times(-\infty, \infty)$ as long as the Riemann problems in Step 2 are solvable.

\section{4. $B V$-Stability}

In this section, we estimate the approximate solutions $W\left(U^{h}(x, y)\right)$ in the total variation norm and prove that the total variation of the approximate solutions $W\left(U^{h}(x, y)\right)$ in $y$, for any fixed $x$, is uniformly bounded with respect to the mesh length $h$. We measure the total variation of approximate solutions by using the sum of the absolute values of the strengths of waves in the solution of each Riemann problem in Step 2 as in Section 3.

We define a weighted $l_{1}-$ norm

$$
\|v\|_{1}=\left|v_{1}\right|+\left|v_{2}\right|+\left|v_{3}\right|+M\left|v_{4}\right|+\left|v_{5}\right| \quad \text { for a vector } v=\left(v_{1}, v_{2}, v_{3}, v_{4}, v_{5}\right) \in \mathbb{R}^{5},
$$

where $M>0$ is a constant to be determined later.

We define another norm

$$
\|v\|=\left|v_{1}\right|+\left|v_{2}\right|+\left|v_{3}\right|+\left|v_{4}\right| \quad \text { for a vector } v=\left(v_{1}, v_{2}, v_{3}, v_{4}\right) \in \mathbb{R}^{4} .
$$

Let $U_{g} \equiv(u, v, p, \rho)$ and $W_{g} \equiv\left(\rho u, \rho u^{2}+p, \rho u v, \rho u\left(\bar{h}+\frac{u^{2}+v^{2}}{2}\right)\right)$ denote the first four components of $U$ and $W$, respectively.

\subsection{Interaction estimates on the non-reacting step}

The interaction estimate for (2.2) is similar to the argument for Proposition 3.1 in [2]. 


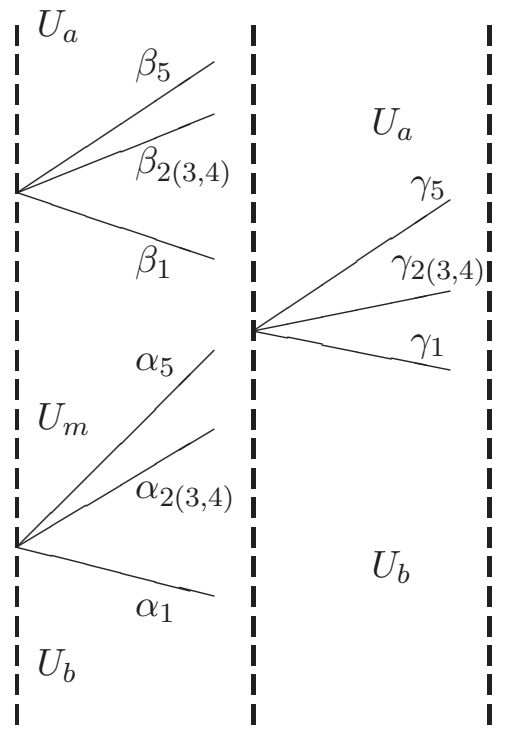

Fig. 7. Weak wave interaction

Lemma 4.1. Suppose that $U_{b}, U_{m}$, and $U_{a}$ are three states in a small neighborhood $O_{\varepsilon}\left(U_{+}\right)$with $\left\{U_{b}, U_{m}\right\}=\left(\alpha_{1}, \alpha_{2}, \alpha_{3}, \alpha_{4}, \alpha_{5}\right),\left\{U_{m}, U_{a}\right\}=\left(\beta_{1}, \beta_{2}, \beta_{3}, \beta_{4}, \beta_{5}\right),\left\{U_{b}, U_{a}\right\}=\left(\gamma_{1}, \gamma_{2}, \gamma_{3}, \gamma_{4}, \gamma_{5}\right)$. Then

$$
\gamma_{i}=\alpha_{i}+\beta_{i}+O(1) \Delta(\alpha, \beta),
$$

where $\Delta(\alpha, \beta)=\left|\alpha_{5}\right|\left(\left|\beta_{1}\right|+\left|\beta_{2}\right|+\left|\beta_{3}\right|+\left|\beta_{4}\right|\right)+\left|\beta_{1}\right|\left(\left|\alpha_{2}\right|+\left|\alpha_{3}\right|+\left|\alpha_{4}\right|\right)+\sum_{j=1,5} \Delta_{j}(\alpha, \beta)$ with

$$
\Delta_{j}(\alpha, \beta)= \begin{cases}0, & \alpha_{j} \geq 0 \text { and } \beta_{j} \geq 0 \\ \left|\alpha_{j}\right|\left|\beta_{j}\right|, & \text { otherwise. }\end{cases}
$$

\subsection{Estimates on the reacting step}

For convenience, we use $\tilde{U}$ to denote the value of $U$ before reaction, while $U$ after reaction. That is,

$$
W(U(x, y))=W(\tilde{U}(x, y))+G(\tilde{U}(x, y)) \tau,
$$

where $\tau=x-k h$ and $k h \leq x<(k+1) h$.

Lemma 4.2. Let

$$
\boldsymbol{\sigma}=\left(\sigma_{1}, \sigma_{2}, \sigma_{3}, \sigma_{4}, \sigma_{5}\right)=B\left(W\left(\tilde{U}_{b}\right), W\left(\tilde{U}_{a}\right)\right)
$$

be the vector of signed wave strengths in the solution of the Riemann problem with Riemann data $\left(W\left(\tilde{U}_{b}\right), W\left(\tilde{U}_{a}\right)\right)$. Let

$$
\Gamma\left(W\left(\tilde{U}_{b}\right), \boldsymbol{\sigma}, h\right)=B\left(W\left(U_{b}\right), W\left(U_{a}\right)\right),
$$

where $W\left(U_{b}\right)=W\left(\tilde{U}_{b}\right)+G\left(\tilde{U}_{b}\right) h$ and $W\left(U_{a}\right)=W\left(\tilde{U}_{a}\right)+G\left(\tilde{U}_{a}\right) h$. Then

$$
\Gamma\left(W\left(\tilde{U}_{b}\right), \boldsymbol{\sigma}, h\right)=\boldsymbol{\sigma}+O\left(\|\boldsymbol{\sigma}\|_{1}\right) h .
$$

Lemma 4.2 implies that the increasing of the total variation of the fractional-step approximate solutions is at no more than an exponential rate.

Lemma 4.3. $\left\|\Gamma\left(W\left(\tilde{U}_{b}\right), \boldsymbol{\sigma}, h\right)-\left(\boldsymbol{\sigma}+\frac{\partial \Gamma}{\partial h}\left(W\left(\tilde{U}_{b}\right), \boldsymbol{\sigma}, 0\right) h\right)\right\|_{1} \leq C\|\boldsymbol{\sigma}\|_{1} \frac{h^{2}}{2}$. 
Lemma 4.3 shows that we can estimate the increase in the total variation for the reacting step by calculating the first derivatives of the solution operator for the Riemann problem.

The proof of Lemmas 4.2-4.3 can be found in [1].

In particular, for (1.1)-(1.5), we need to analyze the reacting step, which takes the form:

$$
W\left(U^{h}(x, y)\right)=W\left(U_{0}^{h}(x, y)\right)+G\left(U_{0}^{h}(x, y)\right) \tau,
$$

where all the quantities $\rho_{0}^{h}, \rho^{h}$, etc. are evaluated at $(k h+\tau, y)$ and $\tau=x-k h$. More precisely, it takes the form:

$$
\begin{aligned}
\rho^{h} u^{h} & =\rho_{0}^{h} u_{0}^{h}, \\
\rho^{h}\left(u^{h}\right)^{2}+p^{h} & =\rho_{0}^{h}\left(u_{0}^{h}\right)^{2}+p_{0}^{h}, \\
\rho^{h} u^{h} v^{h} & =\rho_{0}^{h} u_{0}^{h} v_{0}^{h}, \\
\left(\rho^{h} E^{h}+p^{h}\right) u^{h} & =\left(\rho_{0}^{h} E_{0}^{h}+p_{0}^{h}\right) u_{0}^{h}+q \rho_{0}^{h} z_{0}^{h} \phi\left(T_{0}^{h}\right) \tau, \\
\rho^{h} u^{h} Z^{h} & =\rho_{0}^{h} u_{0}^{h} Z_{0}^{h}-\rho_{0}^{h} Z_{0}^{h} \phi\left(T_{0}^{h}\right) \tau .
\end{aligned}
$$

We need to estimate the change in $(u, v, p, \rho, z, T)$ due to the reaction step.

First, we have

$$
\left(T^{h}-T_{0}^{h}\right)(k h+\tau, y)=\frac{\partial T}{\partial w_{4}} q \rho_{0}^{h} Z_{0}^{h} \phi\left(T_{0}^{h}\right) \tau=\frac{(\gamma-1)\left(\left(u_{0}^{h}\right)^{2}-R T_{0}^{h}\right)}{R \rho_{0}^{h} u_{0}^{h}\left(\left(u_{0}^{h}\right)^{2}-\gamma R T_{0}^{h}\right)} q \rho_{0}^{h} Z_{0}^{h} \phi\left(T_{0}^{h}\right) \tau .
$$

Since $u^{2}>c^{2}=\frac{\gamma p}{\rho}=\gamma R T$, then $T^{h}(x, y) \geq T_{0}^{h}(x, y)$, which shows that the temperature $T$ does not decrease due to the reaction.

Second, from the fifth equation: $Z^{h}-Z_{0}^{h}=-\frac{Z_{0}^{h} \phi\left(T_{0}^{h}\right) \tau}{u_{0}^{h}}$. Since $\phi(T)$ is assumed to be Lipschitz continuous, nonnegative, and increasing, there exists a constant $\Phi_{1}>0$ such that

$$
Z^{h}-Z_{0}^{h} \leq-Z_{0}^{h} \Phi_{1} \tau \text {. }
$$

Then we conclude

$$
Z^{h} \leq Z_{0}^{h}\left(1-\Phi_{1} \tau\right) \leq Z_{0}^{h} e^{-\Phi_{1} \tau}, \quad 0 \leq \tau<h
$$

According to the scheme and using the induction, we can actually obtain

$$
Z_{0}^{h} \leq\left\|Z_{0}\right\|_{\infty} e^{-\Phi_{1} k h}, \quad k h \leq x<(k+1) h .
$$

Third, from the first three equations, we know that $u^{h}=\frac{\rho_{0}^{h} u_{0}^{h}}{\rho^{h}}, v^{h}=v_{0}^{h}$, and $p^{h}=p_{0}^{h}+$ $\rho_{0}^{h}\left(u_{0}^{h}\right)^{2}-\frac{\rho_{0}^{h}\left(u_{0}^{h}\right)^{2}}{\rho^{h}}$. Substitution of these into the fourth equation, we have

$$
\frac{\gamma+1}{2}\left(\rho_{0}^{h} u_{0}^{h}\right)^{2}\left(\frac{1}{\rho^{h}}\right)^{2}-\gamma\left(\rho_{0}^{h}\left(u_{0}^{h}\right)^{2}+p_{0}^{h}\right) \frac{1}{\rho^{h}}+\left(\frac{\gamma-1}{2}\left(u_{0}^{h}\right)^{2}+\gamma \frac{p_{0}^{h}}{\rho_{0}^{h}}+Z_{0}^{h} O(h)\right)=0 .
$$

Therefore, we obtain

$$
\frac{1}{\rho^{h}}=\frac{\gamma\left(\rho_{0}^{h}\left(u_{0}^{h}\right)^{2}+p_{0}^{h}\right)+\sqrt{\left(\rho_{0}^{h}\left(u_{0}^{h}\right)^{2}-\gamma p_{0}^{h}\right)^{2}+\left(\rho_{0}^{h} u_{0}^{h}\right)^{2} Z_{0}^{h} O(h)}}{(\gamma+1)\left(\rho_{0}^{h} u_{0}^{h}\right)^{2}} .
$$

Using the Taylor expansion, we know

$$
\frac{1}{\rho_{0}^{h}}=\frac{1}{\rho^{h}}+Z_{0}^{h} O(h) .
$$

That is,

$$
\rho^{h}-\rho_{0}^{h}=\left\|Z_{0}\right\|_{\infty} e^{-\Phi_{1} k h} O(h) .
$$

Similar calculations also apply to $u$ and $p$. Therefore, we have 
Lemma 4.4. There are positive constants $C_{0}$ and $\Phi_{1}$ such that

$$
\begin{aligned}
T^{h} \geq T_{0}^{h} & \geq C_{0}>0, \\
u^{h}-u_{0}^{h} & =\left\|Z_{0}\right\|_{\infty} e^{-\Phi_{1} k h} O(h), \\
v^{h}-v_{0}^{h} & =0, \\
p^{h}-p_{0}^{h} & =\left\|Z_{0}\right\|_{\infty} O(h) e^{-\Phi_{1} k h}, \\
\rho^{h}-\rho_{0}^{h} & =\left\|Z_{0}\right\|_{\infty} O(h) e^{-\Phi_{1} k h}, \\
Z^{h} & \leq Z_{0}^{h} e^{-\Phi_{1} \tau}, \quad 0 \leq \tau<h .
\end{aligned}
$$

Furthermore,

$$
Z_{0}^{h} \leq\left\|Z_{0}\right\|_{\infty} e^{-\Phi_{1} k h} O(h), \quad k h \leq x<(k+1) h .
$$

All the quantities are evaluated at $(k h+\tau, y)$ with $\tau=x-k h$.

\subsection{Glimm functional for the fractional-step scheme}

Following Glimm's method [8], we define a functional on the restriction of the approximate solution $W\left(U^{h}\right)$ to certain mesh curves $J$. We define a mesh point to be a point $(x, y)=$ $\left(k h,\left(j+\theta_{k}\right) l\right)$, where $k \in \mathbb{N}$ and $j \in \mathbb{Z}$ such that $j+k$ is even. A mesh curve $J$ is a piecewise linear curve in the $(x, y)$-plane, which successively connects the mesh points $\left(k h,\left(j+\theta_{k}\right) l\right)$ to the mesh points $\left((k \pm 1) h,\left(j+1+\theta_{k \pm 1}\right) l\right)$. We define a partial order on the set of mesh curves by stating that larger curves lie toward larger $x$. We call $J_{2}$ an immediate successor of $J_{1}$ if $J_{2}$ connects the same mesh points as $J_{1}$, except for one mesh point, and if $J_{2}>J_{1}$. Let $J_{k}$ be the unique mesh curve which connects the mesh points on $x=k h$ to the mesh points on $x=(k+1) h$. Note that $J_{k}$ crosses all the waves in the Riemann solutions of $W\left(U_{0}^{h}(x, y)\right)$ in the strip $k h \leq x<(k+1) h$.

We now define a functional $F$ on the set of mesh curves. For any mesh curve $J$, we define

$$
L_{i}(J)=\sum\{|\alpha|: \alpha \text { is the } i \text { th wave crossing } J\} \quad \text { for } 1 \leq i \leq 5 .
$$

Next, we define

$$
L(J)=\sum_{1 \leq i \leq 5, i \neq 4} L_{i}(J)+M L_{4}(J)
$$

and

$$
Q(J)=\sum\{|\alpha||\beta| \text { : both } \alpha \text { and } \beta \text { cross } J \text { and approach each other }\},
$$

where $M>0$ is a constant to be determined as in (4.1).

By standard procedure as in [13], when $\operatorname{TV}\left(U_{0}(\cdot)\right)$ is small enough, we can choose a positive constant $K_{0}$ sufficiently large such that the Glimm functional

$$
F(J)=L(J)+K_{0} Q(J)
$$

is non-increasing in the non-reacting step.

\section{4. $B V$-stability of the reaction step}

We now prove the BV-stability of the approximate solutions during the reaction step. Our total variation bounds imply bounds on the length of $W\left(U^{h}(J)\right)$, but we must also deal with the "drift" of the solution due to the reaction term $G(U)$.

In order to discuss the effect of the exothermic reaction on the functionals $L$ and $Q$, it is convenient to identify a new "mesh curve" $\tilde{J}$, which, as a curve, is the same as a given mesh curve $J$, but upon which the value of $W(U)$ differs from the value of $W(U)$ on $J$ by a single reaction step along all of $J$. We take $\tilde{J}$ to represent the values before the reaction and $J$ to represent the values after the reaction step. 
Lemma 4.5. There is a positive constant $C$ such that

$$
\begin{gathered}
L\left(J_{k}\right) \leq L\left(\tilde{J}_{k}\right)+C q h\left\|w_{5,0}\right\|_{\infty} e^{-\Phi_{1} k h} L\left(\tilde{J}_{k}\right), \\
Q\left(J_{k}\right) \leq Q\left(\tilde{J}_{k}\right)+C q h\left\|w_{5,0}\right\|_{\infty} e^{-\Phi_{1} k h} L\left(\tilde{J}_{k}\right)^{2} .
\end{gathered}
$$

Proof. For simplicity of presentation, we denote $\mathbf{c}=\left(0,0,0,1,-\frac{1}{q}\right)^{\top}, \tilde{\boldsymbol{\sigma}}_{i}=\left(\tilde{\sigma}_{1 i}, \tilde{\sigma}_{2 i}, \tilde{\sigma}_{3 i}, \tilde{\sigma}_{4 i}, \tilde{\sigma}_{5 i}\right)$, $\mathbf{c}_{g}=(0,0,0,1)^{\top}, W\left(\tilde{U}_{i+1}\right)=\Psi\left(\tilde{\boldsymbol{\sigma}}_{i}, W\left(\tilde{U}_{i}\right)\right), B=\left(B_{1}, B_{2}, B_{3}, B_{4}, B_{5}\right)^{\top}$, and $B_{g}=\left(B_{1}, B_{2}, B_{3}, B_{4}\right)^{\top}$.

Let

$$
\Gamma\left(W\left(\tilde{U}_{i}\right), \tilde{\boldsymbol{\sigma}}_{\boldsymbol{i}}, h\right)=B\left(W\left(U_{i}\right), W\left(U_{i+1}\right)\right)
$$

as before, where $W\left(U_{i}\right)=W\left(\tilde{U}_{i}\right)+G\left(\tilde{U}_{i}\right) h$ and $W\left(U_{i+1}\right)=W\left(\tilde{U}_{i+1}\right)+G\left(\tilde{U}_{i+1}\right) h$. Then we have

$$
\begin{aligned}
\frac{\partial \Gamma}{\partial h}\left(W\left(\tilde{U}_{i}\right), \tilde{\boldsymbol{\sigma}}_{i}, 0\right) & =\tilde{\rho}_{i} \tilde{Z}_{i} \phi\left(\tilde{T}_{i}\right) \partial_{1} B q \mathbf{c}+\tilde{\rho}_{i+1} \tilde{Z}_{i+1} \phi\left(\tilde{T}_{i+1}\right) \partial_{2} B q \mathbf{c} \\
& =\tilde{w}_{5, i} \frac{\phi\left(\tilde{T}_{i}\right)}{\tilde{u}_{i}} \partial_{1} B q \mathbf{c}+\tilde{w}_{5, i+1} \frac{\phi\left(\tilde{T}_{i+1}\right)}{\tilde{u}_{i+1}} \partial_{2} B q \mathbf{c} \\
& =\tilde{w}_{5, i}\left(\partial_{1} B \frac{\phi\left(\tilde{T}_{i}\right)}{\tilde{u}_{i}}+\partial_{2} B \frac{\phi\left(\tilde{T}_{i+1}\right)}{\tilde{u}_{i+1}}\right) q \mathbf{c}+\left(\tilde{w}_{5, i+1}-\tilde{w}_{5, i}\right) \partial_{2} B \frac{\phi\left(\tilde{T}_{i+1}\right)}{\tilde{u}_{i+1}} q \mathbf{c}
\end{aligned}
$$

where $\tilde{w}_{5, i}=\tilde{\rho}_{i} \tilde{u}_{i} \tilde{Z}_{i}$ and $\tilde{w}_{5, i+1}=\tilde{\rho}_{i+1} \tilde{u}_{i+1} \tilde{Z}_{i+1}$. Since $Z$ is decoupled from $(u, v, p, \rho)^{\top}$ in the solution of the non-reacting Riemann problem, this means that $\partial_{1} B$ and $\partial_{2} B$ are the block $5 \times 5$ matrices with the upper left $4 \times 4$ block relating to non-reacting gas dynamics. The remaining $1 \times 1$ block contains the derivative of wave strength of $Z$-contact with respect to $\rho u Z$ - the value of this derivative is -1 for $\frac{\partial B_{5}}{\partial w_{5, i}}$ and 1 for $\frac{\partial B_{5}}{\partial w_{5, i+1}}$, since $B_{5}=\rho_{i+1} u_{i+1} Z_{i+1}-\rho_{i} u_{i} Z_{i}=w_{5, i+1}-w_{5, i}$. Then we have

$$
\begin{gathered}
\partial_{1} B q \mathbf{c}=\left(\begin{array}{cc}
\partial_{1 W_{g}} B_{g} & 0 \\
0 & -1
\end{array}\right)\left(\begin{array}{c}
q \mathbf{c}_{g} \\
-1
\end{array}\right)=\left(\begin{array}{c}
\partial_{1 W_{g}} B_{g} q \mathbf{c}_{g} \\
0
\end{array}\right)+(0,0,0,0,1)^{\top}, \\
\partial_{2} B q \mathbf{c}=\left(\begin{array}{cc}
\partial_{2 W_{g}} B_{g} & 0 \\
0 & 1
\end{array}\right)\left(\begin{array}{c}
q \mathbf{c}_{g} \\
-1
\end{array}\right)=\left(\begin{array}{c}
\partial_{2 W_{g}} B_{g} q \mathbf{c}_{g} \\
0
\end{array}\right)-(0,0,0,0,1)^{\top},
\end{gathered}
$$

where $W_{g}=\left(w_{1}, \cdots, w_{4}\right)$. Then

$$
\begin{aligned}
\frac{\partial \Gamma}{\partial h}\left(W\left(\tilde{U}_{i}\right), \tilde{\boldsymbol{\sigma}}_{i}, 0\right)= & \tilde{w}_{5, i}\left[\frac{\phi\left(\tilde{T}_{i}\right)}{\tilde{u}_{i}}\left(\begin{array}{c}
\partial_{1 W_{g}} B_{g} q \mathbf{c}_{g} \\
1
\end{array}\right)+\frac{\phi\left(\tilde{T}_{i+1}\right)}{\tilde{u}_{i+1}}\left(\begin{array}{c}
\partial_{2 W_{g}} B_{g} q \mathbf{c}_{g} \\
-1
\end{array}\right)\right] \\
& +\left(\tilde{w}_{5, i+1}-\tilde{w}_{5, i}\right) \frac{\phi\left(\tilde{T}_{i+1}\right)}{\tilde{u}_{i+1}}\left(\begin{array}{c}
\partial_{2 W_{g}} B_{g} q \mathbf{c}_{g} \\
-1
\end{array}\right) .
\end{aligned}
$$

Thus, the first four components of (4.25) have the form:

$$
\begin{aligned}
\frac{\partial \Gamma_{g}}{\partial h}\left(W\left(\tilde{U}_{i}\right), \tilde{\boldsymbol{\sigma}}_{i}, 0\right)= & \tilde{w}_{5, i}\left[\partial_{1 W_{g}} B_{g} q \mathbf{c}_{g} \frac{\phi\left(\tilde{T}_{i}\right)}{\tilde{u}_{i}}+\partial_{2 W_{g}} B_{g} q \mathbf{c}_{g} \frac{\phi\left(\tilde{T}_{i+1}\right)}{\tilde{u}_{i+1}}\right] \\
& +\left(\tilde{w}_{5, i+1}-\tilde{w}_{5, i}\right) \partial_{2 W_{g}} B_{g} q \mathbf{c}_{g} \frac{\phi\left(\tilde{T}_{i+1}\right)}{\tilde{u}_{i+1}} \\
= & \tilde{w}_{5, i} A\left(W\left(\tilde{U}_{i}\right), \tilde{\boldsymbol{\sigma}}_{i}\right)+\left(\tilde{w}_{5, i+1}-\tilde{w}_{5, i}\right) \partial_{2 W_{g}} B_{g} q \mathbf{c}_{g} \frac{\phi\left(\tilde{T}_{i+1}\right)}{\tilde{u}_{i+1}}
\end{aligned}
$$

where

$$
A\left(W\left(\tilde{U}_{i}\right), \tilde{\boldsymbol{\sigma}}_{i}\right)=\partial_{1 W_{g}} B_{g} q \mathbf{c}_{g} \frac{\phi\left(\tilde{T}_{i}\right)}{\tilde{u}_{i}}+\partial_{2 W_{g}} B_{g} q \mathbf{c}_{g} \frac{\phi\left(\tilde{T}_{i+1}\right)}{\tilde{u}_{i+1}}
$$


It is easy to see that, if $\tilde{\boldsymbol{\sigma}}_{g, i}=\left(\tilde{\sigma}_{1, i}, \tilde{\sigma}_{2, i}, \tilde{\sigma}_{3, i}, \tilde{\sigma}_{4, i}\right)=\mathbf{0}$, then $\tilde{W}_{g, i+1}=\tilde{W}_{g, i}:=W_{g}\left(\tilde{U}_{i}\right)$ and, in particular, $\tilde{T}_{i+1}=\tilde{T}_{i}$. Since $B_{g}\left(\tilde{W}_{g, i}, \tilde{W}_{g, i}\right)$ is the vector of wave strengths for a Riemann problem with equal states,

$$
\left.\partial_{1 W_{g}} B_{g}\right|_{\tilde{\boldsymbol{\sigma}}_{g, i}=0}=\left.\partial_{2 W_{g}} B_{g}\right|_{\tilde{\boldsymbol{\sigma}}_{g, i}=0}=0 .
$$

Therefore, there exists some positive constant $C$ such that the first term in (4.26) can be estimated by

$$
\left\|\tilde{w}_{5, i} A\left(W\left(\tilde{U}_{i}\right), \tilde{\boldsymbol{\sigma}}_{i}\right)\right\| \leq C \tilde{w}_{5, i}\left\|\tilde{\boldsymbol{\sigma}}_{g, i}\right\| q .
$$

We next examine the last term of (4.26), which has the form

$$
\left(\tilde{w}_{5, i+1}-\tilde{w}_{5, i}\right) \partial_{2 W_{g}} B_{g} q \mathbf{c}_{g} \frac{\phi\left(\tilde{T}_{i+1}\right)}{\tilde{u}_{i+1}} .
$$

The fifth component of (4.25) is the equation for the strength of the $Z$-wave. This equation is

$$
\frac{\partial}{\partial h}\left(\tilde{w}_{5, i+1}-\tilde{w}_{5, i}\right)=\tilde{w}_{5, i}\left(\frac{\phi\left(\tilde{T}_{i}\right)}{\tilde{u}_{i}}-\frac{\phi\left(\tilde{T}_{i+1}\right)}{\tilde{u}_{i+1}}\right)-\left(\tilde{w}_{5, i+1}-\tilde{w}_{5, i}\right) \frac{\phi\left(\tilde{T}_{i+1}\right)}{\tilde{u}_{i+1}},
$$

so that

$$
\frac{\partial}{\partial h}\left|\tilde{w}_{5, i+1}-\tilde{w}_{5, i}\right| \leq \tilde{w}_{5, i}\left|\frac{\phi\left(\tilde{T}_{i}\right)}{\tilde{u}_{i}}-\frac{\phi\left(\tilde{T}_{i+1}\right)}{\tilde{u}_{i+1}}\right|-\left|\tilde{w}_{5, i+1}-\tilde{w}_{5, i}\right| \frac{\phi\left(\tilde{T}_{i+1}\right)}{\tilde{u}_{i+1}} .
$$

Thus, the reaction step produces possible increases in the total variation, which are bounded by

$$
C \tilde{w}_{5, i}\left\|\tilde{\boldsymbol{\sigma}}_{g, i}\right\| q h+\left|\tilde{w}_{5, i+1}-\tilde{w}_{5, i}\right| \frac{\phi\left(\tilde{T}_{i+1}\right)}{\tilde{u}_{i+1}}\left\|\partial_{2 W_{g}} B_{g} \mathbf{c}_{g}\right\| q h .
$$

The reaction step also produces a decrease in total variation for the $w_{5}=\rho u Z$ componentthe fifth component of $\frac{\partial \Gamma}{\partial h}\left(W\left(\tilde{U}_{i}\right), \tilde{\boldsymbol{\sigma}}_{i}, 0\right)$-in the amount $\left|\tilde{w}_{5, i+1}-\tilde{w}_{5, i}\right| \frac{\phi\left(\tilde{T}_{i+1}\right)}{\tilde{u}_{i+1}}$. We now use the decrease in the $w_{5}$-component proportional to $\left|\tilde{w}_{5, i+1}-\tilde{w}_{5, i}\right|$. Since $\partial_{2 W_{g}} B_{g}$ is Lipschitz continuous, there exists a upper bound $M$ for $\left\|\partial_{2 g} B_{g}\right\| q$. Thus, the effect of term (4.26) on $\left(\rho u, \rho u^{2}+p, \rho u v, \rho u\left(\bar{h}+\frac{u^{2}+v^{2}}{2}\right)\right)$ of $\frac{\partial \Gamma}{\partial h}\left(W\left(\tilde{U}_{i}\right), \tilde{\boldsymbol{\sigma}}_{i}, 0\right)$ is bounded by $M\left|\tilde{w}_{5, i+1}-\tilde{w}_{5, i}\right| \frac{\phi\left(\tilde{T}_{i+1}\right)}{\tilde{u}_{i+1}} h$, and this increase is offset by a decrease in the term $M\left|\tilde{w}_{5, i+1}-\tilde{w}_{5, i}\right|$.

Thus, the change in $L$ is estimated as follows:

$$
\begin{aligned}
L\left(J_{k}\right)-L\left(\tilde{J}_{k}\right) & =\sum_{1 \leq j \leq 5, j \neq 4}\left(L_{j}\left(J_{k}\right)-L_{j}\left(\tilde{J}_{k}\right)\right)+M\left(L_{4}\left(J_{k}\right)-L_{4}\left(\tilde{J}_{k}\right)\right) \\
& =\sum_{1 \leq j \leq 5, j \neq 4} \sum_{-\infty<i<\infty}\left(\left|\sigma_{j, i}\right|-\left|\tilde{\sigma}_{j, i}\right|\right)+M \sum_{-\infty<i<\infty}\left(\left|\sigma_{4, i}\right|-\left|\tilde{\sigma}_{4, i}\right|\right) \\
\leq & \sum_{-\infty<i<\infty}\left\|\frac{\partial \Gamma}{\partial h}\left(W\left(\tilde{U}_{i}\right), \tilde{\boldsymbol{\sigma}}_{i}, 0\right)\right\|_{1} h \\
\leq & \sum_{-\infty<i<\infty}\left(C q \tilde{w}_{5, i}\left\|\tilde{\boldsymbol{\sigma}}_{g, i}|| h+\left|\tilde{w}_{5, i+1}-\tilde{w}_{5, i}\right| \frac{\phi\left(\tilde{T}_{i+1}\right)}{\tilde{u}_{i+1}} q\right\| \partial_{2 g} B_{g} \mathbf{c}_{g} \| h\right. \\
& \left.+M\left(\tilde{w}_{5, i}\left|\frac{\phi\left(\tilde{T}_{i}\right)}{\tilde{u}_{i}}-\frac{\phi\left(\tilde{T}_{i+1}\right)}{\tilde{u}_{i+1}}\right| h-\left|\tilde{w}_{5, i+1}-\tilde{w}_{5, i}\right| \frac{\phi\left(\tilde{T}_{i+1}\right)}{\tilde{u}_{i+1}} h\right)\right) \\
\leq & C q h\left\|\tilde{w}_{5}\right\|_{\infty} L\left(\tilde{J}_{k}\right) \\
\leq & C q h\left\|w_{5,0}\right\|_{\infty} e^{-\Phi_{1} k h} L\left(\tilde{J}_{k}\right),
\end{aligned}
$$

where we have chosen $M>0$ large enough to make the third inequality hold, and the last inequality comes from Lemma 4.4 . 
Consequently, we have

$$
\begin{aligned}
Q\left(J_{k}\right)-Q\left(\tilde{J}_{k}\right) & =\sum_{A p p}\left(\left|\alpha_{i}\right|\left|\beta_{j}\right|-\left|\tilde{\alpha}_{i}\right|\left|\tilde{\beta}_{j}\right|\right) \\
& =\sum_{A p p}\left(\left|\alpha_{i}\right|\left(\left|\beta_{j}\right|-\left|\tilde{\beta}_{j}\right|\right)+\left|\tilde{\beta}_{j}\right|\left(\left|\alpha_{i}\right|-\left|\tilde{\alpha}_{i}\right|\right)\right) \\
& \leq C\left(L\left(J_{k}\right)-L\left(\tilde{J}_{k}\right)\right) L\left(\tilde{J}_{k}\right) .
\end{aligned}
$$

Therefore,

$$
Q\left(J_{k}\right) \leq Q\left(\tilde{J}_{k}\right)+C q h\left\|w_{5,0}\right\|_{\infty} e^{-\Phi_{1} k h} L\left(\tilde{J}_{k}\right)^{2} \leq Q\left(\tilde{J}_{k}\right)+C q h\left\|w_{5,0}\right\|_{\infty} e^{-\Phi_{1} k h} F\left(\tilde{J}_{k}\right)^{2} .
$$

The proof is completed.

Since

$$
F(J)=L(J)+K_{0} Q(J),
$$

we have actually proved the following lemma.

Lemma 4.6. Let $J_{k}$ be a mesh curve between $x=k h$ and $x=(k+1) h$. Then

$$
F\left(J_{k}\right) \leq F\left(\tilde{J}_{k}\right)\left(1+C q h\left\|w_{5,0}\right\|_{\infty} e^{-\Phi_{1} k h}\left(1+F\left(\tilde{J}_{k}\right)\right)\right),
$$

where $C$ is a constant independent of the mesh lengths $l$ and $h$.

We need to obtain a uniform bound on $F$. First of all, we suppose that such a bound exists, namely, $F\left(\tilde{J}_{k}\right) \leq A$ for some positive constant. Then, by (4.35), we have

$$
F\left(J_{k}\right) \leq F\left(\tilde{J}_{k}\right)\left(1+C q\left\|w_{5,0}\right\|_{\infty} e^{-\Phi_{1} k h}(1+A) h\right) .
$$

Since $F$ is non-increasing in the non-reacting step, $F\left(\tilde{J}_{k}\right) \leq F\left(J_{k-1}\right)$. Then we have

$$
F\left(J_{k}\right) \leq F\left(\tilde{J}_{0}\right) \prod_{j=0}^{k}\left(1+C q\left\|w_{5,0}\right\|_{\infty} d^{j}(1+A) h\right),
$$

where $d=e^{-\Phi_{1} h}$. Using the inequality $\ln (1+x) \leq x$ for $x \geq 0$,

$$
\begin{aligned}
\ln \left(\frac{F\left(J_{k}\right)}{F\left(\tilde{J}_{0}\right)}\right) & \leq \sum_{j=0}^{k} \ln \left(1+C q\left\|w_{5,0}\right\|_{\infty} d^{j}(1+A) h\right) \\
& \leq \sum_{j=0}^{k} C q h\left\|w_{5,0}\right\|_{\infty} d^{j}(1+A) \\
& \leq C q h\left\|w_{5,0}\right\|_{\infty}(1+A) \frac{1}{1-d} .
\end{aligned}
$$

Thus we obtain

$$
F\left(J_{k}\right) \leq F\left(\tilde{J}_{0}\right) \exp \left(\frac{C q h\left\|w_{5,0}\right\|_{\infty}(1+A)}{1-e^{-\Phi_{1} h}}\right) .
$$

The function $f(h)=\frac{h}{1-e^{-\Phi_{1} h}}$ is increasing for $h>0$ and tends to $\frac{1}{\Phi_{1}}$ as $h \rightarrow 0$. Thus, for $h$ sufficiently small, we obtain

$$
F\left(J_{k}\right) \leq F\left(\tilde{J}_{0}\right) \exp \left(\frac{C_{1} q\left\|w_{5,0}\right\|_{\infty}(1+A)}{\Phi_{1}}\right)
$$


where $C_{1}=2 C$. Estimate (4.38) is valid as long as $F\left(\tilde{J}_{k}\right) \leq A$. Since $F\left(\tilde{J}_{k}\right) \leq F\left(J_{k-1}\right)$, the condition required for this result is that

$$
F\left(\tilde{J}_{0}\right) \leq \exp \left(-\frac{C_{1} q\left\|w_{5,0}\right\|_{\infty}(1+A)}{\Phi_{1}}\right) A=: g(A) .
$$

The value of A which maximizes $g(A)$ is $A=\frac{\Phi_{1}}{C_{1} q\left\|w_{5,0}\right\|_{\infty}}$. Thus, our least-restrictive condition on $F\left(\tilde{J}_{0}\right)$ is

$$
F\left(\tilde{J}_{0}\right) \leq \exp \left(-1-\frac{C_{1} q\left\|w_{5,0}\right\|_{\infty}}{\Phi_{1}}\right) \frac{\Phi_{1}}{C_{1}\left\|w_{5,0}\right\|_{\infty}} .
$$

We summarize these estimates with the following lemma.

Lemma 4.7. If $F\left(\tilde{J}_{0}\right)$ satisfies (4.39), then, for all $k \geq 1, F\left(\tilde{J}_{k}\right) \leq A$. In particular, if $F\left(\tilde{J}_{0}\right)$ satisfies (4.40), then

$$
F\left(\tilde{J}_{k}\right) \leq A=\frac{\Phi_{1}}{C_{1} q\left\|w_{5,0}\right\|_{\infty}} \quad \text { for all } k \geq 1
$$

Furthermore, if $F\left(\tilde{J}_{0}\right)$ satisfies (4.40), then

$$
F\left(J_{k}\right) \leq F\left(\tilde{J}_{0}\right) \exp \left(\frac{C_{1} q\left\|w_{5,0}\right\|_{\infty}}{\Phi_{1}}+1\right) \quad \text { for all } k \geq 1 .
$$

Next, we need to estimate the amount that the solution "drifts" from its original base point due to the source term $G(U)$. We use $W\left(U_{0}(-\infty)\right)=\lim _{y \rightarrow-\infty} W\left(U_{0}(y)\right)$ as our base point. From our scheme,

$$
W\left(U^{h}(x, y)\right)=W\left(U_{0}^{h}(x, y)\right)+G\left(U_{0}^{h}(x, y)\right)(x-k h) .
$$

We denote $U_{k}^{\infty}=\lim _{y \rightarrow-\infty} U^{h}((k+1) h-, y)$ and $U_{0}(-\infty)=\lim _{y \rightarrow-\infty} U_{0}(y)$. Then $W\left(U_{0}^{\infty}\right)=$ $W\left(U_{0}(-\infty)\right)+G\left(U_{0}(-\infty)\right) h$ and $W\left(U_{k+1}^{\infty}\right)=W\left(U_{k}^{\infty}\right)+G\left(U_{k}^{\infty}\right) h$ for $k \geq 0$. Since $Z_{0}(-\infty)=0$, $G\left(U_{0}(-\infty)\right)=0$. We deduce that $W\left(U_{k+1}^{\infty}\right)=W\left(U_{k}^{\infty}\right)=W\left(U_{0}(-\infty)\right)$. Therefore, for all $(x, y) \in J_{k}$, we have

$$
\begin{aligned}
\left\|W\left(U^{h}(x, y)\right)-W\left(U_{0}(-\infty)\right)\right\| & \leq\left\|W\left(U^{h}(x, y)\right)-W\left(U_{k}^{\infty}\right)\right\|+\left\|W\left(U_{k}^{\infty}\right)-W\left(U_{0}(-\infty)\right)\right\| \\
& =\left\|W\left(U^{h}(x, y)\right)-W\left(U_{k}^{\infty}\right)\right\| \\
& \leq T V\left(W\left(U^{h}(x, \cdot)\right)\right) \\
& \leq C F\left(J_{k}\right) .
\end{aligned}
$$

In summary, we have established the following theorem.

Theorem 4.1. If $\mathrm{TV}\left(W\left(U_{0}\right)\right)$ is sufficiently small, then the fractional-step Glimm scheme generates the approximate solutions $U^{h}(x, y)$ which exist in the whole domain $\{x \geq 0, y \in \mathbb{R}\}$ and have uniformly bounded total variation in the $y$-direction. Moreover, there is a null set $N \subset \Pi_{k=0}^{\infty}(-1,1)$ such that, for each $\theta \in \Pi_{k=0}^{\infty}(-1,1) \backslash N$, there exists a sequence $h_{i} \rightarrow 0$ so that

$$
U_{\theta}=\lim _{h_{i} \rightarrow 0} U_{h_{i}, \theta}
$$

is an entropy solution to problem (1.8) for system (1.1)-(1.5), where the limit is taken in $L_{\text {loc }}^{1}(\Omega)$. Moreover, $U_{\theta}$ has uniformly bounded total variation in the $y$-direction.

The proof of the convergence part will be given in Section 6 . 


\section{Initial-Boundary Value Problem}

In this section, we are concerned with reacting supersonic flows past Lischitz curved wedges. The problem can be formulated as the initial-boundary value problem for system (1.1)-(1.5) in $\Omega$ with initial data on $\Gamma$ :

$$
\left.(u, v, p, \rho, Z)\right|_{x=0}=\left(u_{0}, v_{0}, p_{0}, \rho_{0}, Z_{0}\right)(y) \equiv U_{0}(y), \quad y \in \mathbb{R},
$$

and boundary condition

$$
(u, v) \cdot \mathbf{n}=0 \quad \text { on } \Gamma,
$$

where

$$
\Omega=\{(x, y): y<g(x), x>0\}, \quad \Gamma=\{(x, y): y=g(x), x>0\},
$$

and $\mathbf{n}(x \pm)=\frac{\left(-g^{\prime}(x \pm), 1\right)}{\sqrt{\left(g^{\prime}(x \pm)\right)^{2}+1}}$ is the outer unit normal vector to $\Gamma$ at the point $x \pm$ (see Fig. 3 ).

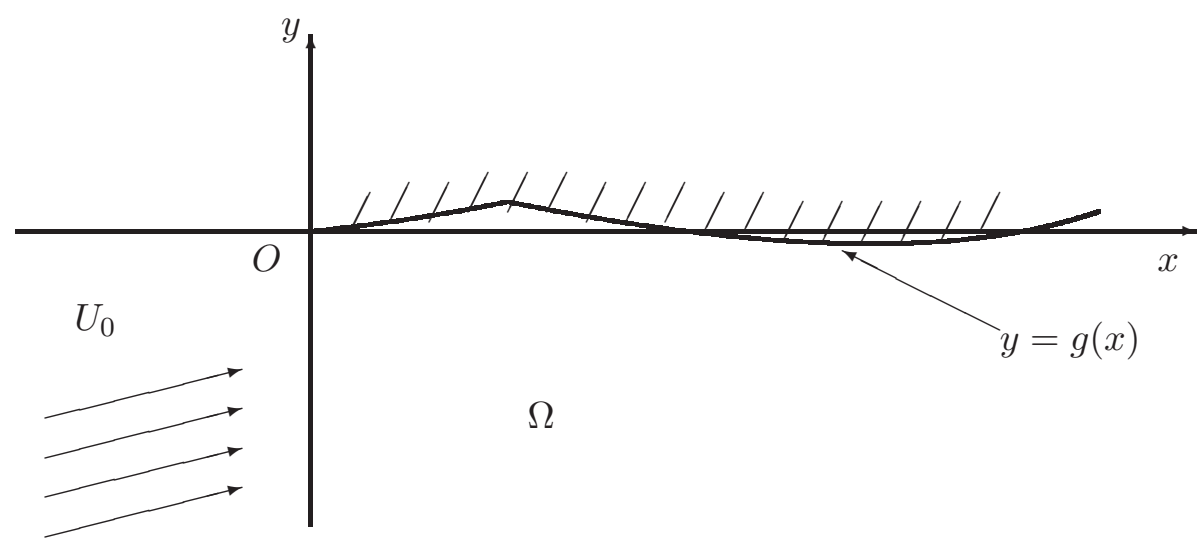

Fig. 3. Supersonic flow past a Lipschitz curved wedge

The assumption for $U_{0}(y):=\left(u_{0}, v_{0}, p_{0}, \rho_{0}, Z_{0}\right)(y)$ is the same as before. The boundary function $y=g(x)$ is a small perturbation of the straight line $y=\frac{v_{0}(-\infty)}{u_{0}(-\infty)} x$ such that $y=g(x)$ is Lipschitz continuous with $g(0)=0, g^{\prime}(0+)=\arctan \left(\frac{v_{0}(-\infty)}{u_{0}(-\infty)}\right)$, and $g^{\prime} \in B V\left(\mathbb{R}^{+} ; \mathbb{R}\right)$.

Without loss of generality, we may assume that

$$
v_{0}(-\infty)=0, \quad Z_{0}(-\infty)=0
$$

The formulation of the initial-boundary value problem is derived from the original physical problem when supersonic flow past a symmetric wedge through the coordinate transformation. For the non-reacting supersonic flow past a straight symmetric wedge, i.e. $g^{\prime}(x)=0$, a plane shock is generated, which is attached to the wedge vertex (see Fig. 4). When the supersonic flow is governed by exothermically reacting steady Euler equations, the attached shock is no longer a plane shock even for the straight wedge, though it can be handled as an approximate shock wave. 


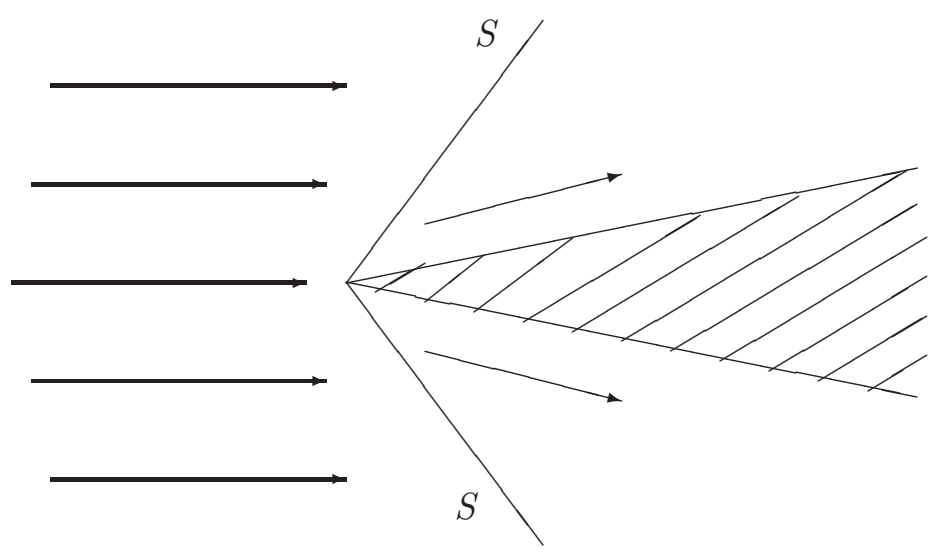

Fig. 4. Non-reacting supersonic flow past a straight wedge

\subsection{Homogeneous initial-boundary value problem}

We first recall some basic properties on the initial-boundary value problem for the homogeneous system (2.2).

\subsubsection{Lateral Riemann problem}

The simple case of problem (1.1) - (1.5) is that $g \equiv 0$. It has been shown in [5] that, if $g \equiv 0$, the homogeneous system (2.2) with initial condition:

$$
\left.(u, v, p, \rho, Z)\right|_{x<0}=\left(u_{-}, v_{-}, p_{-}, \rho_{-}, Z_{-}\right) \equiv U_{-}
$$

yields an entropy solution that consists of the constant states $U_{-}$and $U_{+}:=\left(u_{+}, 0, p_{+}, \rho_{+}, Z_{+}\right)$ with $u_{+}>c_{+}>0$ in the subdomain of $\Omega$, separated by a straight shock-front emanating from the vertex. That is, the state ahead of the shock-front is $U_{-}$, whilst the state behind the shockfront is $U_{+}$(see Figs. 5-6). When the angle between the flow direction of the front state and the wedge boundary at a boundary vertex is larger than $\pi$, the entropy solution contains a rarefaction wave that separates the front state from the back state (see Fig. 6).

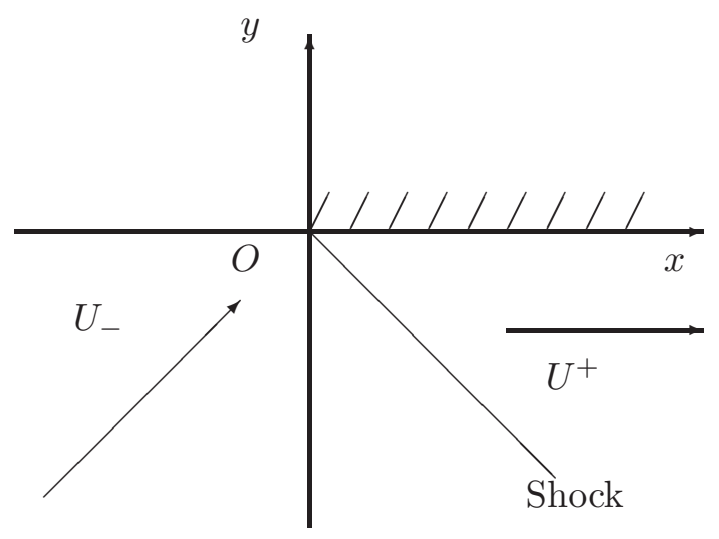

Fig. 5. Unperturbed case when $g \equiv 0$ 


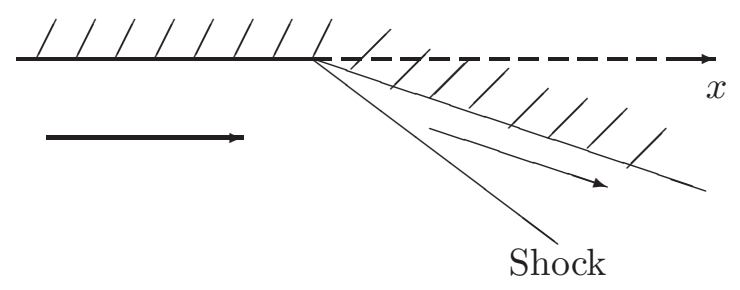

Fig. 6. Lateral Riemann solutions

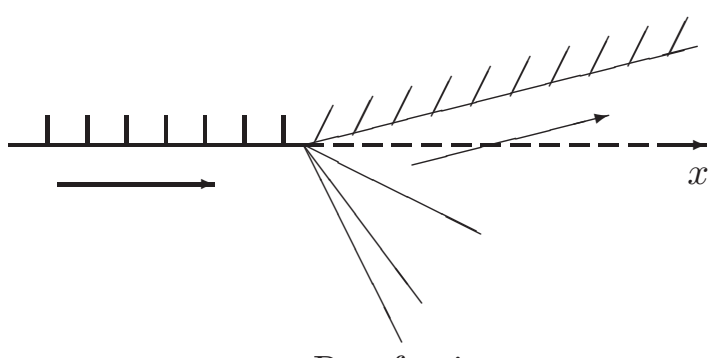

Rarefaction wave

\subsubsection{Riemann problem}

Consider the Riemann problem for (2.2):

$$
\left.U\right|_{x=x_{0}}=U_{-}= \begin{cases}U_{b}, & y<y_{0}, \\ U_{a}, & y>y_{0},\end{cases}
$$

where $U_{a}$ and $U_{b}$ are the constant states which are regarded as the above state and below state with respect to the line $y=y_{0}$, respectively. It is well known that this Riemann problem is solvable if the states $U_{b}$ and $U_{a}$ are close enough.

\subsubsection{Estimates on wave interactions for (2.2)}

The estimates on week wave interactions are the same as in Lemma 4.1

\subsection{Estimates of the reflection on the boundary for system (2.2)}

Following the notation in [18], we denote $\left\{C_{k}\left(a_{k}, b_{k}\right)\right\}_{k=0}^{\infty}$ by the points $\left\{\left(a_{k}, b_{k}\right)\right\}_{k=0}^{\infty}$ in the $(x, y)$-plane with $a_{k+1}>a_{k} \geq 0$. Set

$$
\begin{array}{r}
\omega_{k, k+1}=\arctan \left(\frac{b_{k+1}-b_{k}}{a_{k+1}-a_{k}}\right), \quad \omega_{k}=\omega_{k, k+1}-\omega_{k-1, k}, \quad \omega_{-1,0}=0, \\
\Omega_{k}=\left\{(x, y): x \in\left[a_{k}, a_{k+1}\right), y<b_{k}+\left(x-a_{k}\right) \tan \left(\omega_{k, k+1}\right)\right\}, \\
\Gamma_{k}=\left\{(x, y): x \in\left[a_{k}, a_{k+1}\right), y=b_{k}+\left(x-a_{k}\right) \tan \left(\omega_{k, k+1}\right)\right\},
\end{array}
$$

and the outer unit normal vector to $\Gamma_{k+1}$ :

$$
\mathbf{n}_{k+1}=\frac{\left(-b_{k+1}+b_{k}, a_{k+1}-a_{k}\right)}{\sqrt{\left(b_{k+1}-b_{k}\right)^{2}+\left(a_{k+1}-a_{k}\right)^{2}}}=\left(-\sin \left(\omega_{k, k+1}\right), \cos \left(\omega_{k, k+1}\right)\right) .
$$

We then consider the initial-boundary value problem:

$$
\left\{\begin{array}{l}
\underline{(2.2)} \quad \text { in } \Omega_{k}, \\
\left.U\right|_{x=a_{k}}=\underline{U}, \\
(u, v) \cdot \mathbf{n}_{k}=0 \quad \text { on } \Gamma_{k},
\end{array}\right.
$$

where $\underline{U}$ is a constant state. 


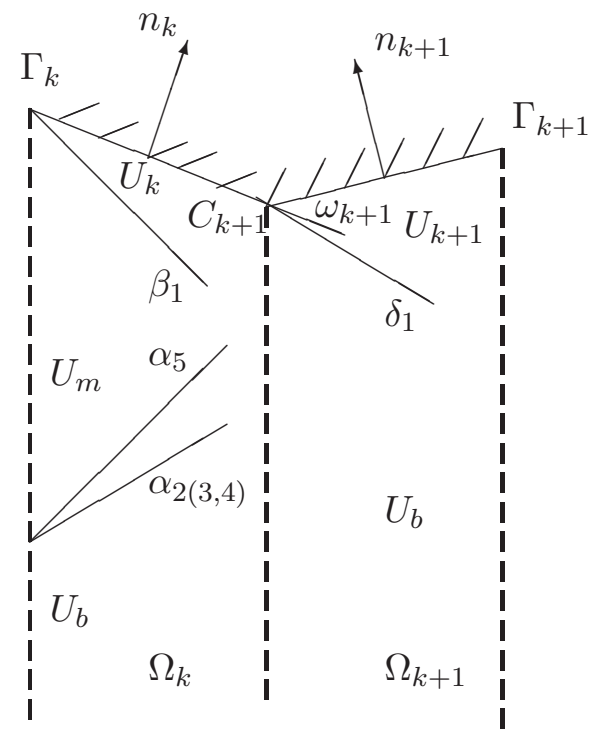

Fig. 8. Weak wave reflections on the boundary.

Lemma 5.1. Let $\left\{U_{b}, U_{m}\right\}=\left(0, \alpha_{2}, \alpha_{3}, \alpha_{4}, \alpha_{5}\right)$ and $\left\{U_{m}, U_{k}\right\}=\left(\beta_{1}, 0,0,0,0\right)$ with

$$
\left(u_{k}, v_{k}\right) \cdot \boldsymbol{n}_{k}=0 .
$$

Then there exists $U_{k+1}$ such that

$$
\left\{U_{b}, U_{k+1}\right\}=\left(\delta_{1}, 0,0,0,0\right) \quad \text { with } \quad\left(u_{k+1}, v_{k+1}\right) \cdot \boldsymbol{n}_{k+1}=0 .
$$

Furthermore,

$$
\delta_{1}=\beta_{1}+K_{b 5} \alpha_{5}+K_{b 4} \alpha_{4}+K_{b 3} \alpha_{3}+K_{b 2} \alpha_{2}+K_{b 0} \omega_{k},
$$

where $K_{b 5}, K_{b 4}, K_{b 3}, K_{b 2}$, and $K_{b 0}$ are $C^{2}$-functions of $\left(\alpha_{5}, \alpha_{4}, \alpha_{3}, \alpha_{2}, \beta_{1}, \omega_{k} ; U_{b}\right)$ satisfying

$$
\left.K_{b 5}\right|_{\omega_{k}=\alpha_{5}=\alpha_{4}=\alpha_{3}=\alpha_{2}=\beta_{1}=0, U_{b}=U_{+}}=1,\left.\quad K_{b i}\right|_{\omega_{k}=\alpha_{5}=\alpha_{4}=\alpha_{3}=\alpha_{2}=\beta_{1}=0, U_{b}=U_{+}}=0, \quad i=2,3,4,
$$

and $K_{b 0}$ is bounded.

The proof of this lemma is similar to Proposition 3.2 in [2].

\subsection{Construction of approximate solutions}

In this section, we develop a modified Glimm difference scheme to construct a family of approximate solutions in consistent with the boundary condition (5.1)-(5.2) and establish their necessary estimates for the initial-boundary value problem for system (1.1)-(1.5) in the corresponding domains $\Omega_{h}$.

We first use the fact that the boundary is a perturbation of the straight wedge:

$$
\sup _{x \geq 0}\left|g^{\prime}(x)\right|<\varepsilon \quad \text { for sufficiently small } \varepsilon>0 .
$$

Let $h>0, l>0$ denote the step-length in the $x$-direction and $y$-direction, respectively. Set $a_{k}:=k h$ and $b_{k}:=y_{k}=g(k h)$ and follow the notations in Section 2.4. Then

$$
m:=\sup _{k>0}\left\{\frac{\left|y_{k}-y_{k-1}\right|}{h}\right\}<\varepsilon .
$$

Define

$$
\Omega_{h}=\bigcup_{k \geq 0} \Omega_{h, k},
$$


where $\Omega_{h, k}=\left\{(x, y): k h \leq x<(k+1) h, \quad y \leq g_{h}(x)\right\}$ with $g_{h}(x)=y_{k}+(x-k h) \tan \left(\omega_{k, k+1}\right)$ when $k h \leq x<(k+1) h$. We also need the Courant-Friedrichs-Lewy type condition:

$$
\max _{1 \leq j \leq 5}\left(\sup _{U \in O_{\varepsilon}\left(U_{+}\right)}\left|\lambda_{j}(U)\right|\right) \leq \frac{l-m h}{2 h} .
$$

Define

$$
a_{k, n}=\left(2 n+1+\theta_{k}\right) l+y_{k},
$$

where $\theta_{k}$ is randomly chosen in $(-1,1)$. Then we choose

$$
P_{k, n}=\left(k h, a_{k, n}\right), \quad k \geq 0, n=0,-1,-2, \cdots,
$$

to be the mesh points and define the approximate solutions $W\left(U^{h}(x, y)\right)$ in $\Omega_{h}$ for any $\theta=$ $\left(\theta_{0}, \theta_{1}, \theta_{2}, \cdots\right)$ in an inductive way.

We denote $T_{k, 0}$ the diamond domain whose vertices are $\left(k h, y_{k}\right),\left(k h,-l+y_{k}\right),((k+1) h,-l+$ $\left.y_{k+1}\right)$, and $\left((k+1) h, y_{k+1}\right)$. For $n \leq-1$, we denote $T_{k, n}$ the diamond whose vertices are $\left(k h,(2 n+1) l+y_{k}\right),\left(k h,(2 n-1) l+y_{k}\right),\left((k+1) h,(2 n-1) l+y_{k+1}\right)$, and $\left((k+1) h,(2 n+1) l+y_{k+1}\right)$.

Now we can define the difference scheme in $\Omega_{h}$, that is, define the global approximate solution $W\left(U^{h}(x, y)\right)$ in $\Omega_{h}$. This can be done by carrying out the following steps inductively, similar to the construction in Section 3.

Assume that $W\left(U^{h}(x, y)\right)$ is defined for $x<k h$. Then we define $W\left(U^{h}(k h+0, y)\right)$ as follows:

We define, for $n \leq-1$,

$$
W\left(U_{0}^{k}\right):=W\left(U^{h}\left(k h-, a_{k, n}\right)\right) \quad \text { for } \quad 2 n l+y_{k} \leq y<2(n+1) l+y_{k},
$$

and

$$
W\left(U^{h}(k h+0, y)\right):=W\left(U_{0}^{k}\right) .
$$

First, we define $W\left(U_{0}^{h}(x, y)\right)$ in $T_{k, 0}$ by solving the following lateral Riemann problem:

$$
\left\{\begin{array}{l}
W\left(U_{k}\right)_{x}+H\left(U_{k}\right)_{y}=0 \quad \text { in } T_{k, 0}, \\
\left.W\left(U_{k}\right)\right|_{x=k h}=W\left(U_{0}^{k}\right), \\
\left(u_{k}, v_{k}\right) \cdot \mathbf{n}_{k}=0 \quad \text { on } \Gamma_{k} .
\end{array}\right.
$$

We can obtain the above lateral Riemann solution $W\left(U_{k}\right)$ in $T_{k, 0}$ and define

$$
W\left(U_{0}^{h}\right)=W\left(U_{k}\right) \quad \text { in } T_{k, 0} .
$$

Second, we solve the following Riemann problem in each diamond $T_{k, n}$ for $n \leq-1$ :

$$
\left\{\begin{array}{l}
W\left(U_{k}\right)_{x}+H\left(U_{k}\right)_{y}=0 \quad \text { in } T_{k, n}, \\
\left.W\left(U_{k}\right)\right|_{x=k h}=W\left(U_{0}^{k}\right),
\end{array}\right.
$$

to obtain the Riemann solution $W\left(U_{k}\right)$ in $T_{k, n}$ and define

$$
W\left(U_{0}^{h}\right)=W\left(U_{k}\right) \quad \text { in } T_{k, n}, n \leq-1 .
$$

Finally, we use the Glimm fractional-step operator to obtain the desired approximate solutions:

$$
W\left(U^{h}(x, y)\right)=W\left(U_{0}^{h}(x, y)\right)+G\left(U_{0}^{h}(x, y)\right)(x-k h) \quad \text { for } k h \leq x<(k+1) h .
$$

In this way, we have constructed the approximate solution $W\left(U^{h}(x, y)\right)$ globally, provided that we can obtain a uniform bound of the approximate solutions. To achieve this, we establish the total variation of $W\left(U^{h}(x, y)\right)$ on a class of space-like curves.

As before, for the mesh curves $J$ in $x>0$, we give the following definition: 


\section{Definition 5.1.}

$$
\begin{aligned}
& L_{0}(J)=\sum\left\{\omega\left(C_{k}\right): C_{k} \in \Omega_{J}\right\}, \\
& L_{j}(J)=\sum\left\{\left|\alpha_{j}\right|: \alpha_{j} \text { cross } J\right\}, \quad j=1,2,3,4,5, \\
& L(J)=K^{*} L_{0}(J)+L_{1}(J)+K^{*}\left(L_{2}(J)+L_{3}(J)+L_{4}(J)+L_{5}(J)\right), \\
& Q(J)=\sum\left\{\left|\alpha_{i}\right|\left|\beta_{j}\right|: \text { both } \alpha_{i} \text { and } \beta_{j} \text { crossing } J \text { and approaching }\right\}, \\
& F(J)=L(J)+K Q(J),
\end{aligned}
$$

where $K>0$ is determined later, $\Omega_{J}$ is the set of the corner points $C_{k}$ with $k \geq 0$ :

$$
\Omega_{J}=\left\{C_{k}: C_{k} \in J \cap \partial \Omega_{h}, C_{k}=(k h, g(k h)), k \geq 0\right\},
$$

and $K^{*}$ is a positive constant that satisfies $K^{*}>\max _{2 \leq i \leq 5} K_{b i}+1$.

Next, we estimate the functional $F$. To do this, let $I$ and $J$ be two $k$-mesh curves for some $k>0$ such that $J$ is an immediate successor to $I$, and let $\Lambda$ be the diamond between $I$ and $J$. Due to the location of $\Lambda$, two cases are to be considered:

(i) Case $\Lambda \subset \Omega_{h}$ : If $\alpha$ and $\beta$ are the waves entering $\Lambda$, we define

$$
Q(\Lambda)=\sum\left|\alpha_{i}\right|\left|\beta_{j}\right|
$$

where the sum is taken over all the pairs for which the $i$-wave from $\alpha$ and $j$-wave from $\beta$ are approaching;

(ii) Case $\Lambda \cap \partial \Omega_{h} \neq \emptyset$ : Let $\Omega_{J}=\Omega_{I} \backslash\left\{C_{k}\right\}$ with $C_{k}=\left(k h, y_{k}\right)$ for some $k \geq 0$, let $I=I_{0} \cup I^{\prime}$ and $J=I_{0} \cup J^{\prime}$ such that $\partial \Lambda=I^{\prime} \cup J^{\prime}$, and let $\beta_{1}$ and $\alpha_{i}$ be the 1-wave and $i$-wave respectively crossing $I^{\prime}$ with $\alpha_{i}$ lying below $\beta_{1}$ on $I$, where $i=2,3,4,5$. In addition, by the construction of approximate solutions, let $\delta_{1}$ be the weak 1-wave crossing $J^{\prime}$ (see Fig. 9 below).

Define

$$
E_{h, \theta}(\Lambda)= \begin{cases}\omega_{k}+\sum_{i=2}^{5}\left|\alpha_{i}\right| & \text { if } \Lambda \cap \partial \Omega_{h} \neq \emptyset, \\ Q(\Lambda) & \text { if } \Lambda \subset \Omega_{h} .\end{cases}
$$

\subsection{Estimates of the non-reacting step involving the boundary}

By choosing a suitable constant $K$, we now prove that the Glimm-type functional $F$ is non-increasing in the non-reacting step.

Theorem 5.1. Suppose that the wedge function $g(x)$ satisfies (5.11), and $I$ and $J$ are two mesh curves such that $J$ is an immediate successor of $I$. Then there exist constants $\varepsilon>0$ and $K>0$ such that, if $F(I) \leq \varepsilon$, then

$$
F(J) \leq F(I)-\frac{1}{4} E_{h, \theta}(\Lambda) .
$$

Proof. We divide our proof into two cases depending on the location of the diamond.

Case 1 (interior weak-weak interaction): $\Lambda$ lies in the interior of $\Omega_{h}$. Denote $Q(\Lambda)=\Delta(\alpha, \beta)$ as defined in Lemma 2.1. Then, for some constant $M>0$,

$$
L(J)-L(I) \leq\left(1+4 K^{*}\right) M Q(\Lambda) .
$$


Since $L\left(I_{0}\right)<\varepsilon$ from $F(I)<\varepsilon$, we have

$$
\begin{aligned}
Q(J)-Q(I) & =\left(Q\left(I_{0}\right)+\sum_{i=1}^{5} Q\left(\gamma_{i}, I_{0}\right)\right)-\left(Q\left(I_{0}\right)+Q(\Lambda)+\sum_{i=1}^{5} Q\left(\alpha_{i}, I_{0}\right)+\sum_{i=1}^{5} Q\left(\beta_{i}, I_{0}\right)\right) \\
& \leq Q\left(M Q(\Lambda), I_{0}\right)-Q(\Lambda) \\
& \leq\left(M L\left(I_{0}\right)-1\right) Q(\Lambda) \\
& \leq-\frac{1}{2} Q(\Lambda) .
\end{aligned}
$$

Hence, by choosing a suitably large $K$, we obtain

$$
F(J)-F(I) \leq\left(\left(1+4 K^{*}\right) M-\frac{K}{2}\right) Q(\Lambda) \leq-\frac{1}{4} Q(\Lambda) .
$$

Case 2 (near the boundary): $\Lambda$ touches the approximate boundary $\partial \Omega_{h}$. Then $\Omega_{J}=$ $\Omega_{I} \backslash\left\{C_{k}\right\}$ for certain $k$.

Let $\delta_{1}$ be the weak 1 -wave going out of $\Lambda$ through $J^{\prime}$, and let $\beta_{1}, \alpha_{2}, \alpha_{3}, \alpha_{4}$, and $\alpha_{5}$ be the weak waves entering $\Lambda$ through $I^{\prime}$, as shown in Fig. 9. Then

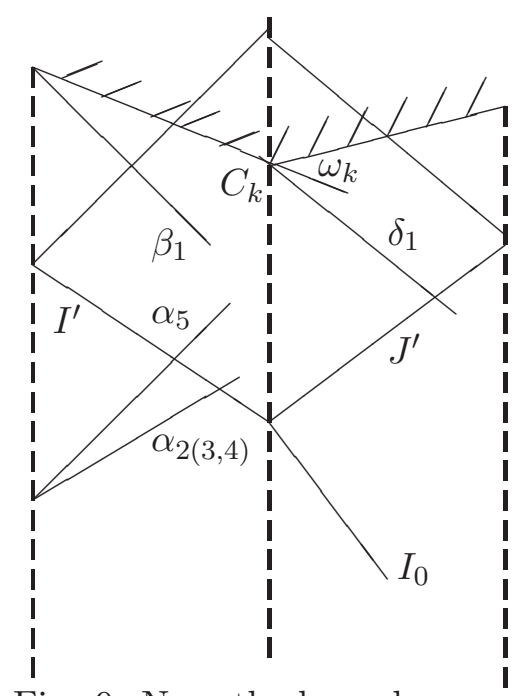

Fig. 9. Near the boundary.

$$
\begin{gathered}
L_{0}(J)-L_{0}(I)=-\left|\omega_{k}\right|, \\
L_{i}(J)-L_{i}(I)=\sum_{\gamma_{i} \operatorname{cross} I_{0}}\left|\gamma_{i}\right|-\left(\left|\alpha_{i}\right|+\sum_{\gamma_{i} \operatorname{cross} I_{0}}\left|\gamma_{i}\right|\right)=-\left|\alpha_{i}\right|, \quad i=2,3,4,5, \\
L_{1}(J)-L_{1}(I)=\left(\left|\delta_{1}\right|+\sum_{\gamma_{1} \operatorname{cross} I_{0}}\left|\gamma_{1}\right|\right)-\left(\left|\beta_{1}\right|+\sum_{\gamma_{1} \operatorname{cross} I_{0}}\left|\gamma_{1}\right|\right) \\
=\left|\delta_{1}\right|-\left|\beta_{1}\right| \\
\leq \sum_{i=2}^{5}\left|K_{b i}\right|\left|\alpha_{i}\right|+\left|K_{b 0}\right|\left|\omega_{k}\right|
\end{gathered}
$$


where the last step is from Lemma 5.1. Thus,

$$
\begin{aligned}
L(J)-L(I) & \leq\left(\left|K_{b 0}\right|-K^{*}\right)\left|\omega_{k}\right|+\sum_{i=2}^{5}\left(\left|K_{b i}\right|-K^{*}\right)\left|\alpha_{i}\right| \\
& \leq-\left(\left|\omega_{k}+\sum_{i=2}^{5}\right| \alpha_{i} \mid\right),
\end{aligned}
$$

since $K^{*}>\max K_{b i}+1$ for $i=1,2,3,4,5$. Moreover, we have

$$
\begin{aligned}
Q(J)-Q(I) & =\left(Q\left(I_{0}\right)+Q\left(\delta_{1}, I_{0}\right)\right)-\left(Q\left(I_{0}\right)+Q\left(\beta_{1}, I_{0}\right)+\sum_{i=2}^{5} Q\left(\alpha_{i}, I_{0}\right)+\left|\beta_{1}\right| \sum_{i=2}^{5}\left|\alpha_{i}\right|\right) \\
& \leq\left(\sum_{i=2}^{5}\left|K_{b i}\right|\left|\alpha_{i}\right|+\left|K_{b 0}\right|\left|\omega_{k}\right|\right) L\left(I_{0}\right) .
\end{aligned}
$$

Then we obtain

$$
\begin{aligned}
F(J)-F(I) & =(L(J)-L(I))+K(Q(J)-Q(I)) \\
& \leq-\left(\left|\omega_{k}\right|+\sum_{i=2}^{5}\left|\alpha_{i}\right|\right)+K\left(\sum_{i=2}^{5}\left|K_{b i}\right|\left|\alpha_{i}\right|+\left|K_{b 0}\right|\left|\omega_{k}\right|\right) L\left(I_{0}\right) \\
& \leq-\frac{1}{4}\left(\left|\omega_{k}\right|+\sum_{i=2}^{5}\left|\alpha_{i}\right|\right)
\end{aligned}
$$

since we can choose $\varepsilon$ sufficiently small. The proof is completed.

\subsection{Estimates of the reacting step involving the boundary}

We first consider the change of the wave strength before and after reaction near the boundary. We denote by $\left(\tilde{U}_{b}, \tilde{U}_{*}\right)$ and $\tilde{\beta}_{1}$ the two states and wave strength before reaction, respectively, while by $\left(U_{b}, U_{*}\right)$ and $\beta_{1}$ after reaction, respectively (see Figure 10). According to the boundary condition, we have
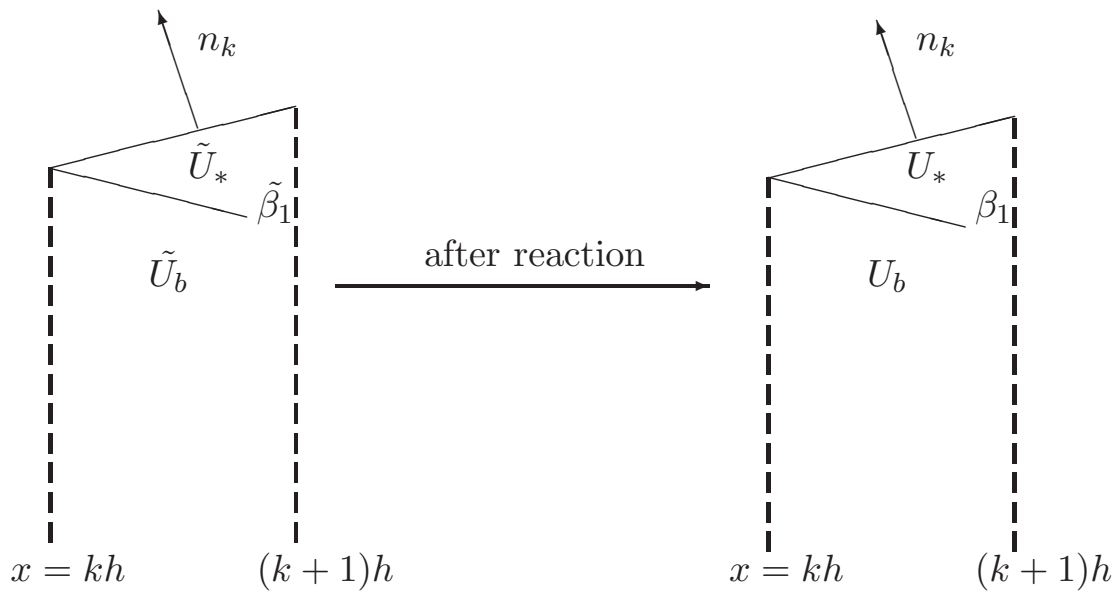

Fig. 10. Change of wave strength near the boundary 
where

$$
W\left(U_{b}(x, y)\right)=W\left(\tilde{U}_{b}(x, y)\right)+G\left(\tilde{U}_{b}(x, y)\right)(x-k h), \quad k h \leq x<(k+1) h,
$$

and

$$
W\left(U_{*}(x, y)\right)=W\left(\tilde{U}_{*}(x, y)\right)+G\left(\tilde{U}_{*}(x, y)\right)(x-k h), \quad k h \leq x<(k+1) h .
$$

From Lemma 4.4. $U_{b}-\tilde{U}_{b}=\left\|Z_{0}\right\|_{\infty} e^{-\Phi_{1} k h} O(h)$ and $U_{*}-\tilde{U}_{*}=\left\|Z_{0}\right\|_{\infty} O(h) e^{-\Phi_{1} k h}$. Therefore, we obtain

$$
\beta_{1}-\tilde{\beta}_{1}=\left\|Z_{0}\right\|_{\infty} O(h) e^{-\Phi_{1} k h} .
$$

As to the inner part, if we perform the same procedure as in the case of the Cauchy problem, we can obtain a similar estimate:

$$
L\left(J_{k}\right)-L\left(\tilde{J}_{k}\right) \leq C h\left\|w_{5,0}\right\|_{\infty} e^{-\Phi_{1} k h} L\left(\tilde{J}_{k}\right) .
$$

Combining these two parts together, we have the following global estimate:

$$
L\left(J_{k}\right)-L\left(\tilde{J}_{k}\right) \leq C h\left\|w_{5,0}\right\|_{\infty} e^{-\Phi_{1} k h}\left(L\left(\tilde{J}_{k}\right)+1\right) .
$$

Therefore, we can do the same procedure as before to establish

Theorem 5.2. If $\mathrm{TV}\left(W\left(U_{0}\right)\right)+\mathrm{TV}\left(g^{\prime}\right)$ is sufficiently small, then the fractional-step Glimm scheme generates the approximate solutions $U^{h}(x, y)$ which exist in the whole domain $\Omega$ and have uniformly bounded total variation in the $y$-direction. Moreover, there is a null set $N \subset$ $\Pi_{k=0}^{\infty}(-1,1)$ such that, for each $\theta \in \Pi_{k=0}^{\infty}(-1,1) \backslash N$, there exist a sequence $h_{i} \rightarrow 0$ so that

$$
U_{\theta}=\lim _{h_{i} \rightarrow 0} U_{h_{i}, \theta}
$$

is a weak solution to problem (5.1)-(5.2) for system (1.1)-(1.5), where the limit is taken in $L_{l o c}^{1}(\Omega)$. Moreover, $U_{\theta}$ has uniformly bounded total variation in the $y$-direction.

The proof of the convergence part of Theorem 5.2 is in Section 6 .

\section{Convergence to Entropy Solutions}

In this section we show that the limit function of the approximate solutions is an entropy solution to the Cauchy problem (1.8) -(1.9) and the initial-boundary value problem (5.1) $-(5.2)$ for system (1.1) $-(1.5)$.

Let $d \theta_{k}$ denote the uniform probability measure on $(-1,1)$, and let $d \theta$ denote the induced product probability measure for the random sample $\left\{\theta_{k}\right\}_{k=1}^{\infty}$ in the Cartesian product space $\mathscr{A}=\prod_{k=1}^{\infty}(-1,1)$.

\section{Theorem 6.1. Suppose that}

(i) The sequence $U^{h}(x, y)$ is constructed by using the Glimm fractional-step scheme with the random sample $\left\{\theta_{k}\right\}_{k=0}^{\infty}$ chosen from $\mathscr{A}$.

(ii) There exist a null set $\mathscr{N} \subset \mathscr{A}$ such that, for $\left\{\theta_{k}\right\} \subset \mathscr{A}-\mathscr{N}$, the sequence $U^{h}(x, y)$ is uniformly bounded in $L^{\infty}$ and converges pointwise a.e. to the function $U(x, y)$. 
Then the function $U(x, y)$ is an entropy solution of the corresponding problem (1.8)-(1.9), or problem (5.1) -(5.2), for system (1.1) -(1.5). That is, for any convex entropy pair $(\eta, q)$ with respect to $W(U)$, the following inequality

$$
\eta(W(U))_{x}+q(W(U))_{y} \leq \nabla_{W} \eta(W(U)) G(U)
$$

holds in the sense of distributions in $\mathbb{R}^{2}$ for problem (1.8)-(1.9) and in $\Omega$ including the boundary for problem (5.1) - (5.2), which means that

$$
\begin{array}{r}
\iint_{\Omega}\left(\left(\eta(W(U)) \phi_{x}+q(W(U)) \phi_{y}+\nabla_{W} \eta(W(U)) G(U) \phi\right) d x d y\right. \\
+\int_{-\infty}^{\infty} \eta\left(W\left(U_{0}(y)\right)\right) \phi(0, y) d y \geq 0
\end{array}
$$

where $\phi(x, y) \geq 0$ : for the Cauchy problem (1.8) with $\Omega=\mathbb{R}^{2}$ and $\phi \in C_{0}^{\infty}\left(\mathbb{R}^{2}\right)$; and for the initial-boundary value problem (5.1) (5.2), either $\phi \in C_{0}^{\infty}(\Omega)$, or $\phi \in C_{0}^{\infty}\left(\mathbb{R}^{2}\right)$ and $(\eta, q)=$ $\alpha(W(U))(u, v)$ for any smooth function $\alpha(W)$ of $W$.

Proof. We focus our proof on the initial-boundary value problem (1.8)-(1.9), since the proof for the Cauchy problem (5.1)-(5.2) is simpler.

We define

$$
\begin{aligned}
L(\theta, h, \phi)= & \iint_{\Omega_{h}}\left(\eta\left(W\left(U^{h}\right)\right) \phi_{x}+q\left(W\left(U^{h}\right)\right) \phi_{y}+\nabla_{W} \eta\left(W\left(U^{h}\right)\right) G\left(U^{h}\right) \phi\right) d x d y \\
& +\int_{-\infty}^{0} \eta\left(W\left(U_{0}(y)\right)\right) \phi(0, y) d y .
\end{aligned}
$$

We only need to prove that $\lim _{h \rightarrow 0} L(\theta, h, \phi) \geq 0$ for $\left\{\theta_{k}\right\} \subset \mathscr{A}-\mathscr{N}$.

Since $U_{0}^{h}(x, y)$ is an entropy solution of conservation laws $W(U)_{x}+H(U)_{y}=0$ in the domain $\Omega_{h, k}$, then

$$
\begin{aligned}
& \iint_{\Omega_{h, k}}\left(\eta\left(W\left(U_{0}^{h}\right)\right) \phi_{x}+q\left(W\left(U_{0}^{h}\right)\right) \phi_{y}\right) d x d y+\int_{-\infty}^{y_{k}} \eta\left(W\left(U_{0}^{h}(k h+0, y)\right)\right) \phi(k h, y) d y \\
& -\int_{-\infty}^{y_{k+1}} \eta\left(W\left(U_{0}^{h}((k+1) h-, y)\right)\right) \phi((k+1) h-, y) d y \geq 0,
\end{aligned}
$$

that is,

$$
\begin{aligned}
& \iint_{\Omega_{h, k}}\left(\eta\left(W\left(U_{0}^{h}\right)\right) \phi_{x}+q\left(W\left(U_{0}^{h}\right)\right) \phi_{y}\right) d x d y+\int_{-\infty}^{0} \eta\left(W\left(U_{0}^{h}\left(k h+0, y+y_{k}\right)\right)\right) \phi\left(k h, y+y_{k}\right) d y \\
& -\int_{-\infty}^{0} \eta\left(W\left(U_{0}^{h}\left((k+1) h-, y+y_{k+1}\right)\right)\right) \phi\left((k+1) h-, y+y_{k+1}\right) d y \geq 0 .
\end{aligned}
$$

Here we have used the fact that $\left(u_{0}^{h}, v_{0}^{h}\right) \cdot n_{k}=0$ on the boundary, and the assumptions for $(\eta, q)$ and $\phi$. Since $W\left(U^{h}(x, y)\right)=W\left(U_{0}^{h}(x, y)\right)+G\left(U_{0}^{h}(x, y)\right)(x-k h)$, then

$$
\begin{aligned}
& \eta\left(W\left(U^{h}(x, y)\right)\right)-\eta\left(W\left(U_{0}^{h}(x, y)\right)\right) \\
& =\nabla_{W} \eta\left(W\left(U_{0}^{h}(x, y)\right)\right) G\left(U_{0}^{h}(x, y)\right)(x-k h)+\varepsilon(x-k h ; x, y)(x-k h)
\end{aligned}
$$


for some function $\varepsilon(s ; x, y)$, which converges uniformly to 0 as $s \rightarrow 0$. Multiplying the above equation by $\phi_{x}$ on both sides and integrating on $\Omega_{h, k}$, we have

$$
\begin{aligned}
& \iint_{\Omega_{h, k}}\left(\eta\left(W\left(U^{h}\right)\right)-\eta\left(W\left(U_{0}^{h}\right)\right)\right) \phi_{x} d x d y \\
& =\iint_{\Omega_{h, k}} \varepsilon(x-k h ; x, y)(x-k h) \phi_{x} d x d y+\iint_{\Omega_{h, k}} \nabla_{W} \eta\left(W\left(U_{0}^{h}(x, y)\right)\right) G\left(U_{0}^{h}(x, y)\right)(x-k h) \phi_{x} d x d y \\
& =\iint_{\Omega_{h, k}} \varepsilon(x-k h ; x, y)(x-k h) \phi_{x} d x d y-\iint_{\Omega_{h, k}} \nabla_{W} \eta\left(W\left(U_{0}^{h}(x, y)\right)\right) G\left(U_{0}^{h}(x, y)\right) \phi_{x} d x d y \\
& \quad-\iint_{\Omega_{h, k}} \frac{\partial}{\partial x}\left(\nabla_{W} \eta\left(W\left(U_{0}^{h}(x, y)\right)\right) G\left(U_{0}^{h}(x, y)\right)\right)(x-k h) \phi d x d y \\
& \quad+\int_{\Gamma_{k}} \nabla_{W} \eta\left(W\left(U_{0}^{h}(x, y)\right)\right) G\left(U_{0}^{h}(x, y)\right)(x-k h) \phi n_{k}^{1} d s \\
& \left.\quad+h \int_{-\infty}^{0} \nabla_{W} \eta\left(W\left(U_{0}^{h}\left((k+1) h-, y+y_{k+1}\right)\right)\right) G\left(U_{0}^{h}(k+1) h-, y+y_{k+1}\right)\right) \\
& \left.\quad \times \phi(k+1) h-, y+y_{k+1}\right) d y .
\end{aligned}
$$

Therefore, we use equation (6.6) to obtain

$$
\begin{aligned}
& \iint_{\Omega_{h, k}} \eta\left(W\left(U^{h}\right)\right) \phi_{x} \\
& \geq-\iint_{\Omega_{h, k}}\left(q\left(W\left(U_{0}^{h}\right)\right) \phi_{y}+\nabla_{W} \eta\left(W\left(U_{0}^{h}(x, y)\right)\right) G\left(U_{0}^{h}(x, y)\right) \phi\right) d x d y \\
& \quad+\iint_{\Omega_{h, k}} \varepsilon(x-k h ; x, y)(x-k h) \phi_{x} d x d y \\
& \quad+\int_{-\infty}^{0}\left(\eta\left(W\left(U_{0}^{h}\left((k+1) h-, y+y_{k+1}\right)\right)\right) \phi\left((k+1) h, y+y_{k+1}\right)\right. \\
& \quad-\int_{\Omega_{h, k}} \nabla_{W} \eta\left(W\left(U_{0}^{h}(x, y)\right)\right) G\left(U_{0}^{h}(x, y)\right) \phi d x d y \\
& \quad-\iint_{\Omega_{h, k}} \frac{\partial}{\partial x}\left(\nabla_{W} \eta\left(W\left(U_{0}^{h}(x, y)\right)\right) G\left(U_{0}^{h}(x, y)\right)\right)(x-k h) \phi d x d y \\
& \quad+\int_{\Gamma_{k}} \nabla_{W} \eta\left(W\left(U_{0}^{h}(x, y)\right)\right) G\left(U_{0}^{h}(x, y)\right)(x-k h) \phi n_{k}^{1} d s \\
& \left.\quad+h \int_{-\infty}^{0} \nabla_{W} \eta\left(W\left(U_{0}^{h}\left((k+1) h-, y+y_{k+1}\right)\right)\right) G\left(U_{0}^{h}(k+1) h-, y+y_{k+1}\right)\right) \\
& \left.\quad \times \phi(k+1) h-, y+y_{k+1}\right) d y .
\end{aligned}
$$


Summing over $k$, we have

$$
L(\theta, h, \phi) \geq \mathscr{A}(\theta, h, \phi)+\sum_{k=0}^{\infty} \mathscr{B}_{k}(\theta, h, \phi)+\sum_{k=0}^{\infty} \mathscr{C}_{k}(\theta, h, \phi)+\sum_{k=0}^{\infty} \mathscr{D}_{k}(\theta, h, \phi),
$$

where

$$
\begin{aligned}
\mathscr{A}(\theta, h, \phi)= & \sum_{k=0}^{\infty} \mathscr{A}_{k}(\theta, h, \phi), \\
\mathscr{A}_{0}(\theta, h, \phi)= & \int_{-\infty}^{0}\left(\eta\left(W\left(U_{0}(y)\right)\right)-\eta\left(W\left(U_{0}^{h}(0, y)\right)\right)\right) \phi(0, y) d y, \\
\mathscr{A}_{k}(\theta, h, \phi)= & \int_{-\infty}^{0}\left(\eta\left(W\left(U_{0}^{h}\left(k h-, y+y_{k}\right)\right)\right)-\eta\left(W\left(U_{0}^{h}\left(k h+0, y+y_{k}\right)\right)\right) \phi\left(k h, y+y_{k}\right) d y\right. \\
& +h \int_{-\infty}^{0} \nabla_{W} \eta\left(W\left(U_{0}^{h}\left((k+1) h-, y+y_{k+1}\right)\right)\right) \times \\
& \left.\left.\left.\left.+\iint_{\Omega_{h, k}}\left(\nabla_{W} \eta\left(W\left(U^{h}\right)\right) G\left(U^{h}\right)-\nabla_{W} \eta(W+1) h-, y+y_{k+1}\right)\right) \phi(k+1) h-, y+y_{k+1}^{h}\right)\right) G\left(U_{0}^{h}\right)\right) \phi d x d y \\
& +\iint_{\Omega_{h, k}} \varepsilon(x-k h ; x, y)(x-k h) \phi_{x} d x d y, \\
\mathscr{B}_{k, k}(\theta, h, \phi) & \left.\quad\left(W\left(U^{h}\right)\right)-q\left(W\left(U_{0}^{h}\right)\right) \phi_{y}\right) d x d y \\
& \iint_{\Omega_{h, k}} \frac{\partial}{\partial x}\left(\nabla_{W} \eta\left(W\left(U_{0}^{h}(x, y)\right)\right) G\left(U_{0}^{h}(x, y)\right)\right)(x-k h) \phi d x d y, \\
\mathscr{D}_{k}(\theta, h, \phi)= & \int \nabla_{\Gamma_{k}} \eta\left(W\left(U_{0}^{h}(x, y)\right)\right) G\left(U_{0}^{h}(x, y)\right)(x-k h) \phi n_{k}^{1} d s . \\
\mathscr{C}_{k}(\theta, h, \phi) &
\end{aligned}
$$

The proof for each component converging to zero as $h$ tends to zero is similar to [1], and we omit here. This completes the proof.

\section{Asymptotic Behavior involving the Boundary}

Let $\theta \in \Pi_{k=0}^{\infty}(-1,1) \backslash \mathcal{N}$ be equidistributed. To determine the asymptotic behavior of the solution $U(x, y)$, we need further estimates on $U_{h, \theta}$.

Lemma 7.1. There exists a constant $M_{1}>0$, independent of $U_{h, \theta}, \theta$ and $h$, such that

$$
\sum_{\Lambda} E_{h, \theta}(\Lambda) \leq M_{1}
$$

where the summation is over all the diamonds.

Proof. First, from the conclusion of the non-reacting step, i.e. Theorem 5.1, we know

$$
F(J)-F(I) \leq-\frac{1}{4} E_{h, \theta}(\Lambda)
$$


where $J$ is an immediate successor of $I$. Then we conclude

$$
F\left(\tilde{J}_{k}\right)-F\left(J_{k-1}\right) \leq-\frac{1}{4} \sum_{k-1}^{k+1} E_{h, \theta}(\Lambda),
$$

where the summation is over all the diamonds between $x=(k-1) h$ and $x=(k+1) h$.

Then we know from the reacting step that

$$
F\left(J_{k}\right)-F\left(\tilde{J}_{k}\right) \leq C h\left\|w_{5,0}\right\|_{\infty} e^{-\Phi_{1} k h}\left(F\left(\tilde{J}_{k}\right)+1\right)^{2} .
$$

Combine these two steps together and sum for $k$ from 1 to $\infty$ to obtain

$$
\begin{aligned}
\sum_{k=1}^{\infty} \sum_{k-1}^{k+1} E_{h, \theta}(\Lambda) & \leq C F\left(J_{0}\right)+\sum_{k=1}^{\infty} C h\left\|w_{5,0}\right\|_{\infty} e^{-\Phi_{1} k h}\left(F\left(\tilde{J}_{k}\right)+1\right)^{2} \\
& \leq C\left(F\left(J_{0}\right)+\left\|w_{5,0}\right\|_{\infty}\right)<\infty .
\end{aligned}
$$

The proof is completed.

Moreover, let $\Gamma_{g}=\cup_{k=0}^{\infty} \bar{\Lambda}_{k, 0}$, where $\Lambda_{k, 0}$ is the diamond centered at $C_{k}$, and let $L_{h, \theta}\left(\Gamma_{g}\right)$ be the summation of the strength of waves leaving $\Gamma_{g}$. Then we have

Lemma 7.2. There exists a constant $M_{2}$ independent of $U_{h, \theta}, h$, and $\theta$ such that

$$
L_{h, \theta}\left(\Gamma_{g}\right) \leq M_{2} \sum_{\Lambda} E_{h, \theta}(\Lambda)
$$

This can be obtained by employing Lemmas 5.1-5.2 and (5.36) and by taking the summation of them over $\Gamma_{g}$.

For $i=2,3,4,5$, let $L_{i}(a-)$ be the amount of all $i$-waves in $U_{\theta}$ crossing the line $x=a$ for any $a>0$. Also, let $\tilde{L}_{i}^{h, \theta}(a)$ and $L_{i}^{h, \theta}(a)$ denote the amount of $i$-waves before reaction and after reaction, respectively, in $U_{h, \theta}$ crossing the line $x=a$ for any $a>0$.

Lemma 7.3. $L_{i}(x-) \rightarrow 0$ as $x \rightarrow \infty$, for $i=2,3,4,5$.

Proof. In fact, for $k h \leq x<(k+1) h$,

$$
\tilde{L}_{i}^{h, \theta}(x)-L_{i}^{h, \theta}(x) \leq L\left(J_{k}\right)-L\left(\tilde{J}_{k}\right) \leq C h\left\|w_{5,0}\right\|_{\infty} e^{-\Phi_{1} k h}\left(L\left(\tilde{J}_{k}\right)+1\right) .
$$

Then, by Lemmas 7.1-7.2, we can perform the same procedure as in [19] and conclude this result.

Next, we study the asymptotic behavior of the trace of $U$ on the boundary. To this end, from Lemmas 7.1-7.2, we can first deduce

Lemma 7.4. Let

$$
B_{h, \theta}(x)=U_{h, \theta}\left(x, g_{h}(x)\right) .
$$

Then there exists a constant $M>0$ depending only on the system such that

$$
\operatorname{TV}\left\{B_{h, \theta} ;[0, \infty)\right\} \leq M .
$$

Then, by Lemma 7.4, we can choose a subsequence $\left\{h_{i_{l}}\right\}$ of $\left\{h_{i}\right\}$ so that

$$
B_{h_{i_{l}}, \theta} \rightarrow B_{\theta}
$$

in $L_{l o c}^{1}([0, \infty))$ as $h_{i_{l}} \rightarrow 0$ for some $B_{\theta} \in L^{\infty}$. From the construction of approximate solutions, we have 
Lemma 7.5. Let $B_{\theta}$ be given by (7.9). Then

$$
B_{\theta} \in B V([0, \infty))
$$

and

$$
B_{\theta}(x-) \cdot\left(-g^{\prime}(x-), 1,0,0,0\right)=0 .
$$

Proof. Since

$$
\begin{aligned}
& B_{h_{i_{l}}, \theta}(x-) \cdot\left(-g_{h_{i_{l}}}^{\prime}(x-), 1,0,0,0\right) \\
& =\left(B_{h_{i_{l}}, \theta}(x-)-\tilde{B}_{h_{i_{l}}, \theta}(x-)\right) \cdot\left(-g_{h_{i_{l}}}^{\prime}(x-), 1,0,0,0\right)+\tilde{B}_{h_{i_{l}}, \theta}(x-) \cdot\left(-g_{h_{i_{l}}}^{\prime}(x-), 1,0,0,0\right),
\end{aligned}
$$

the first term on the right-hand side tend to 0 as $h_{i_{l}} \rightarrow 0$, while the second term equals to 0 . Then we conclude the result.

Moreover, we can determine the asymptotic behavior of the traces of $U_{\theta}$ on $\partial \Omega$ as follows.

Lemma 7.6. There holds the following

$$
\sup _{\hat{\lambda} x \leq y \leq g(x)}\left|U_{\theta}(x-, y)-B_{\theta}(x-)\right| \rightarrow 0 \quad \text { as } x \rightarrow \infty
$$

for any $\hat{\lambda} \in\left(\sup \lambda_{1}, \inf g^{\prime}\right)$.

Proof. Notice that

$$
\begin{aligned}
& \sup _{\hat{\lambda} x \leq y \leq g(x)}\left|U_{\theta}(x-, y)-B_{\theta}(x-)\right| \\
& \leq \sup _{\hat{\lambda} x \leq y \leq g(x)}\left|U_{\theta}(x-, y)-\tilde{U}_{\theta}(x-, y)\right|+\sup _{\hat{\lambda} x \leq y \leq g(x)}\left|\tilde{U}_{\theta}(x-, y)-B_{\theta}(x-)\right| .
\end{aligned}
$$

By Lemma 7.3, the first term on the right-hand side tends to zero. In the same way as in [19], the second term also tends to zero. The proof is completed.

From Lemmas 7.3 and 7.6, it follows that

Lemma 7.7. Let

$$
B_{\theta}(\infty)=\lim _{x \rightarrow \infty} B_{\theta}(x-)
$$

and let

$$
g^{\prime}(\infty)=\lim _{x \rightarrow \infty} g_{+}^{\prime}(x)
$$

Then

$$
\lim _{x \rightarrow \infty} \sup _{\hat{\lambda} x \leq y \leq g(x)}\left|\lambda_{1}\left(U_{\theta}(x-, y)\right)-\lambda_{1}\left(B_{\theta}(x-)\right)\right|=0
$$

and

$$
B_{\theta}(\infty) \cdot\left(-g^{\prime}(\infty), 1\right)=0
$$

Repeating the argument as in [11] and by Lemmas 7.3 and 7.7, we can prove

Lemma 7.8. Let $U_{\infty}=\lim _{y \rightarrow-\infty} U_{0}(y)$ for the initial data $U_{0}(y)$ at $x=0$.

(i) If $\lambda_{1}\left(B_{\theta}(\infty)\right)>\lambda_{1}\left(U_{\infty}\right)$, then

$$
B_{\theta}(\infty) \in R_{1}^{+}\left(U_{\infty}\right)
$$


(ii) If $\lambda_{1}\left(B_{\theta}(\infty)\right) \leq \lambda_{1}\left(U_{\infty}\right)$, then

$$
B_{\theta}(\infty) \in S_{1}^{-}\left(U_{\infty}\right)
$$

Therefore, the equation

$$
\Phi\left(0,0,0,0, \alpha_{\infty} ; U_{\infty}\right)=B_{\theta}(\infty)
$$

has a unique solution $\alpha_{\infty}$.

Considering the geometry of the boundary and performing the same way as in [19], we can obtain

Lemma 7.9. Suppose that $\left|g^{\prime}(\infty)\right|$ is small, then

(i) If $g^{\prime}(\infty)<0$, then $\lambda_{1}\left(B_{\theta}(\infty)\right)>\lambda_{1}\left(U_{\infty}\right)$;

(ii) If $g^{\prime}(\infty)=0$, then $\lambda_{1}\left(B_{\theta}(\infty)\right)=\lambda_{1}\left(U_{\infty}\right)$;

(iii) If $g^{\prime}(\infty)>0$, then $\lambda_{1}\left(B_{\theta}(\infty)\right)<\lambda_{1}\left(U_{\infty}\right)$.

By carrying out the same arguments as in [19] and employing the above lemmas, we finally have the asymptotic behavior of entropy solutions.

Theorem 7.1. Suppose that $\operatorname{TV}\left(U_{0}\right)+\mathrm{TV}\left(g^{\prime}\right)$ is sufficiently small.

(i) If $g^{\prime}(\infty)<0$, then there exists a 1 -shock which approaches to the shock wave with $\left(\alpha_{\infty}, 0,0,0,0\right)$ both in strength and speed as $x \rightarrow \infty$; moreover, the total variation of $U_{\theta}$ outside this shock approaches to zero as $x \rightarrow \infty$.

(ii) If $g^{\prime}(\infty)=0$, then $\sup _{y<g(x)}\left|U_{\theta}(x, y)-U_{\infty}\right| \rightarrow 0$ as $x \rightarrow \infty$.

(iii) If $g^{\prime}(\infty)>0$, then the amount of shocks approaches to zero as $x \rightarrow \infty$ and $U(x, y)$ approaches the rarefaction wave with $\left(\alpha_{\infty}, 0,0,0,0\right)$, where $\left(\alpha_{\infty}, 0,0,0,0\right)$ is given in Lemma 7.8 .

\section{Supersonic Reacting Euler Flow past Lipschitz Wedge with Large Angle}

Now we consider the general case when the wedge angle is arbitrary large, but less than the sonic angle. We establish a theory of global existence and asymptotic behavior of entropy solutions for the initial-boundary value problem (5.1) -(5.2) for system (1.1)-(1.5) for which $v_{0}(-\infty)$ is not zero in general.

\subsection{Initial-boundary value problem involving a strong shock}

For the wedge with large vertex angle, as in [2], we choose a suitable coordinate system (by rotation when it is necessary) such that the wedge has the lower boundary $\{y=g(x), x \geq 0\}$ with

$$
g(0)=g^{\prime}(0)=0, \quad g \in C([0, \infty]), \quad g^{\prime} \in \mathrm{BV} .
$$




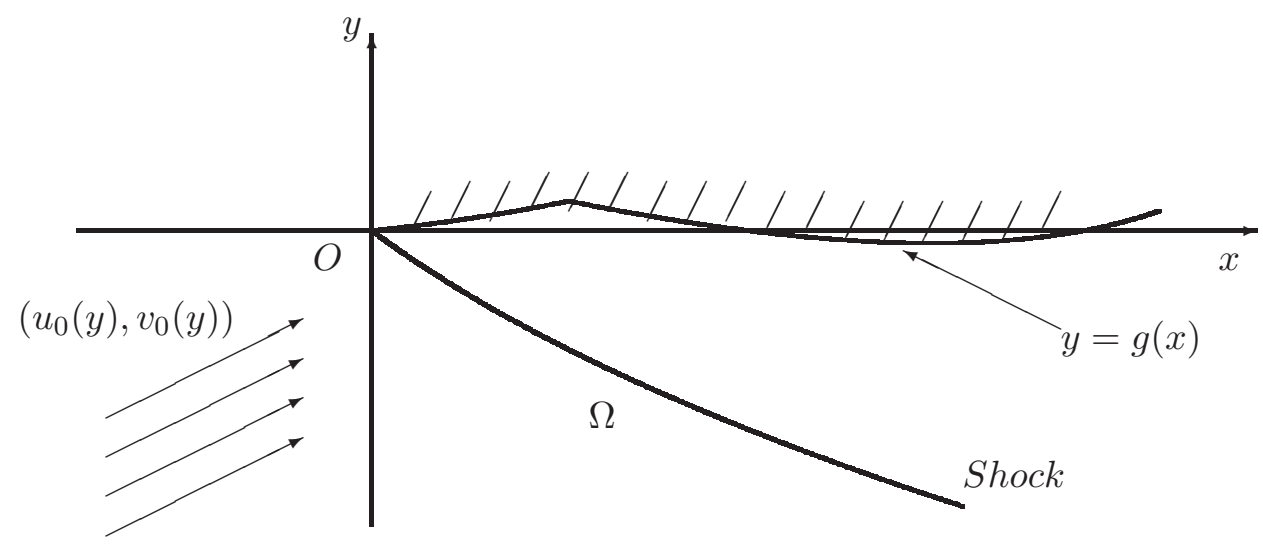

Fig. 11. Initial-boundary problem with large vertex angle

For the non-reaction problem with straight boundary $\{x \geq 0, y \equiv 0\}$ and uniform incoming flow $U_{0}(-\infty)$, if we assume that

$$
0<\arctan \left(\frac{v_{0}(-\infty)}{u_{0}(-\infty)}\right)<\omega_{\text {crit }}
$$

then there exists a supersonic state $U_{+}=\left(u_{+}, 0, p_{+}, \rho_{+}, Z_{+}\right) \in S_{1}\left(U_{0}(-\infty)\right)$ with entropy condition $u_{+}<u_{0}(-\infty)$ such that the corresponding non-reaction problem (2.24)-(2.28) has a shock solution with a leading shock front issuing from the vertex (see Fig. 12).

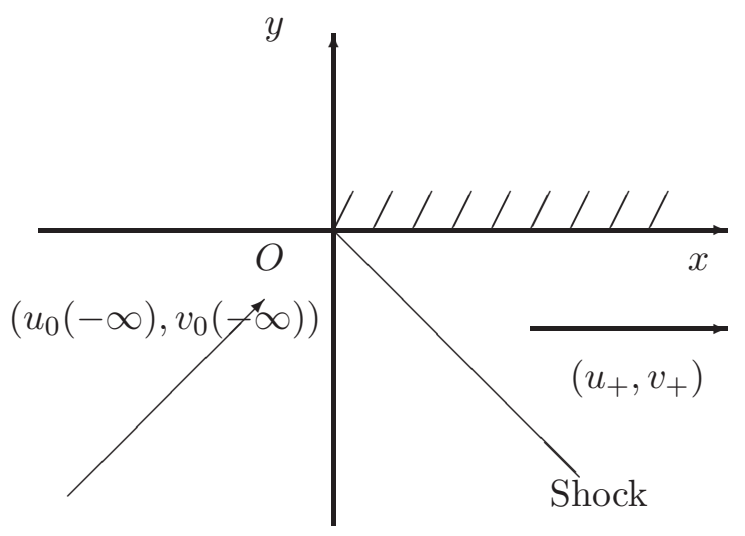

Fig. 12. The background solution for the no-reaction problem

Moreover, there exist $r_{1}>0$ and $r_{2}>0$ such that, for any $U_{1} \in O_{r_{2}}\left(U_{0}(-\infty)\right)$, the shock polar $S_{1}\left(U_{1}\right) \cap O_{r_{1}}\left(U_{+}\right)$can be parameterized by the form

$$
U=D\left(s, U_{1}\right) \quad \text { with } U_{+}=D\left(s, U_{-\infty}\right),
$$

where $s$ is the shock speed.

\subsection{Riemann problem with a strong shock}

To construct the approximate solutions, we need to solve the Riemann problem with a strong shock. 
Lemma 8.1. Let $U_{1} \in O_{r_{1}}\left(U_{0}(-\infty)\right)$ and $U_{2} \in O_{r_{2}}\left(U_{+}\right)$with small positive constants $r_{1}>0$ and $r_{2}>0$. Then the Riemann problem

$$
\left\{\begin{array}{c}
W(U)_{x}+H(U)_{y}=0, \\
\left.U\right|_{x=0}= \begin{cases}U_{1} & y<y_{0}, \\
U_{2} & y>y_{0},\end{cases}
\end{array}\right.
$$

has a unique solution constituted by weak waves $\alpha_{2}, \alpha_{3}, \alpha_{4}, \alpha_{5}$, and a strong shock $s$, that is,

$$
\Psi\left(\alpha_{5}, \alpha_{4}, \alpha_{3}, \alpha_{2}, 0 ; D\left(s, U_{1}\right)\right)=U_{2} .
$$

This lemma can be proved in the same way as in [2] by solving (8.5). Besides the Riemann problem for the interacting weak waves and the fractional steps in the previous sections, we also employ (8.4) for dealing with the interaction between the weak waves and the strong wave. More precisely, we have the following lemma to include the strong shock.

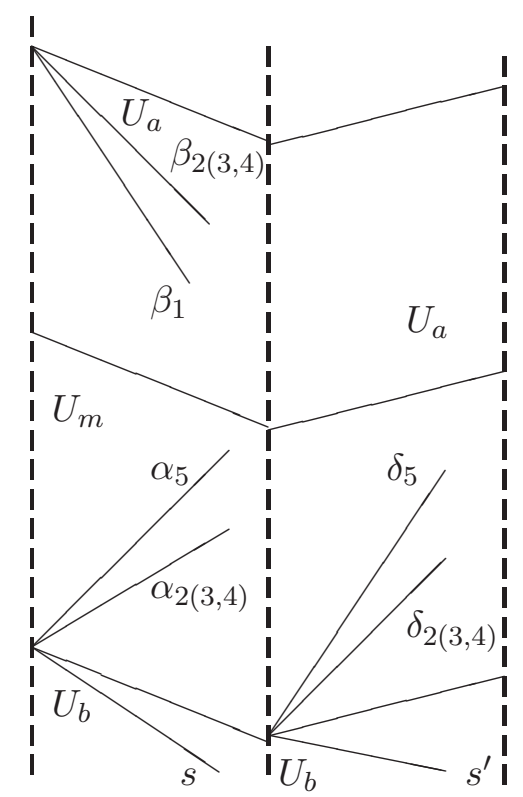

Fig. 13. Interaction with the strong wave below

Lemma 8.2. Suppose that $U_{b} \in O_{r_{1}}\left(U_{0}(-\infty)\right)$ and $U_{a}, U_{m} \in O_{r_{2}}\left(U_{+}\right)$with

$$
\begin{aligned}
& \left\{U_{m}, U_{a}\right\}=\left(\beta_{1}, \beta_{2}, \beta_{3}, \beta_{4}, 0\right), \\
& \left\{U_{b}, U_{m}\right\}=\left(s, \alpha_{2}, \alpha_{3}, \alpha_{4}, \alpha_{5}\right),
\end{aligned}
$$

and

$$
\left\{U_{b}, U_{a}\right\}=\left(s^{\prime}, \delta_{2}, \delta_{3}, \delta_{4}, \delta_{5}\right)
$$

Then

$$
\begin{aligned}
& s^{\prime}=s+K_{s_{1}} \beta_{1}+O(1) \Delta, \\
& \delta_{j}=\alpha_{j}+\beta_{j}+K_{s_{j}} \beta_{1}+O(1) \Delta, \quad j=2,3,4, \\
& \delta_{5}=\alpha_{5}+K_{s_{5}} \beta_{1}+O(1) \Delta,
\end{aligned}
$$

with

$$
\left|K_{s 5}\right|<1, \quad \sum_{j}\left|K_{s j}\right| \leq M \quad \text { for some } M>0
$$


and

$$
\Delta=\left|\alpha_{5}\right|\left(\left|\beta_{2}\right|+\left|\beta_{3}\right|+\left|\beta_{4}\right|\right)
$$

Lemma 8.3. Suppose that

$$
\left\{U_{b}, U_{m}\right\}=\left(\alpha_{1}, \alpha_{2}, \alpha_{3}, \alpha_{4}, \alpha_{5}\right), \quad\left\{U_{m}, U_{a}\right\}=\left(s, \beta_{2}, \beta_{3}, \beta_{4}, \beta_{5}\right),
$$

and

$$
\left\{U_{b}, U_{a}\right\}=\left(s^{\prime}, \delta_{2}, \delta_{3}, \delta_{4}, \delta_{5}\right),
$$

with $U_{b}, U_{m} \in O_{r_{2}}\left(U_{0}(-\infty)\right)$ and $U_{a} \in O_{r_{1}}\left(U_{+}\right)$. Then

$$
s^{\prime}=s+K_{s_{1}} \alpha_{1}+O(1) \sum_{j=1}^{5}\left|\alpha_{j}\right|, \quad \delta_{j}=\beta_{j}+O(1) \sum_{j=1}^{5}\left|\beta_{j}\right| .
$$

Proof. Actually, if we set $\alpha_{j}=0$ for all $j$, then $s^{\prime}=s$ and $\delta_{j}=\beta_{j}$ for all $j$. Then the result follows.

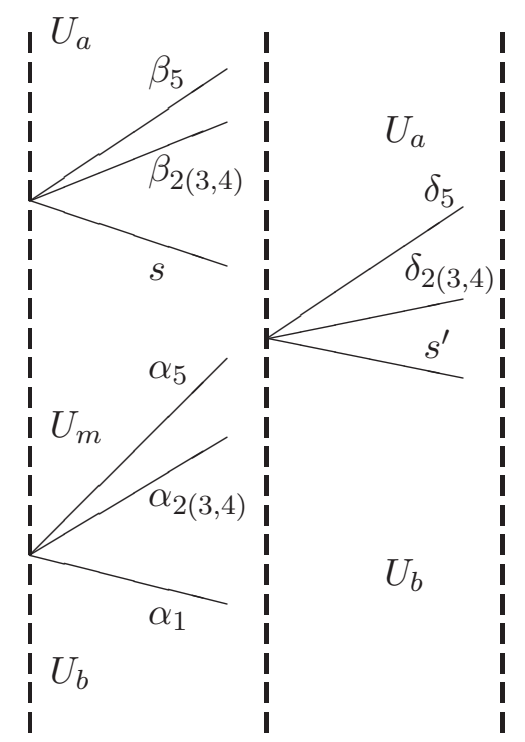

Fig. 14. Interaction with the strong wave above

\subsection{Glimm-type functional involving the strong shock}

We use the same grid points and mesh curves as in the previous sections. For the strip $\Omega_{k}$, we denote the strong shock in $\Omega_{k}$ by $s_{k}$. Without confusion, we also denote its speed by $s_{k}$ and its location by $y=\chi_{k}(x)$.

Let

$$
\Omega_{k+}=\left\{\chi_{k}(x)<y\right\} \cap \Omega_{k}, \quad \Omega_{k-}=\left\{\chi_{k}(x)>y\right\} \cap \Omega_{k} .
$$

For $J_{k}<J<J_{k+1}$, we denote $J_{+}=J \cap \Omega_{k+}$ and $J_{-}=J \cap \Omega_{k-}$. 


\section{Definition 8.1.}

$$
\begin{aligned}
& L_{j}\left(J_{ \pm}\right)=\sum\left\{|\alpha|: \alpha \text { is weak } j \text {-wave crossing } J_{ \pm}\right\} \\
& Q\left(J_{ \pm}\right)=\sum\left\{|\alpha||\beta|: \alpha, \beta \text { are weak waves, approaching and crossing } J_{ \pm}\right\}, \\
& L\left(J_{+}\right)=K_{0}^{*} L_{0}(J)+L_{1}\left(J_{+}\right)+K_{2}^{*} L_{2}\left(J_{+}\right)+K_{3}^{*} L_{3}\left(J_{+}\right)+K_{4}^{*} L_{4}\left(J_{+}\right)+K_{5}^{*} L_{5}\left(J_{+}\right), \\
& L\left(J_{-}\right)=L_{1}\left(J_{-}\right)+K_{2}^{* *} L_{2}\left(J_{-}\right)+K_{3}^{* *} L_{3}\left(J_{-}\right)+K_{4}^{* *} L_{4}\left(J_{-}\right)+K_{5}^{* *} L_{5}\left(J_{-}\right), \\
& F(J)=L\left(J_{+}\right)+K L\left(J_{-}\right)+K^{\prime} Q\left(J_{+}\right)+K K^{\prime \prime} Q\left(J_{-}\right), \\
& F_{s}(J)=\left|s_{J}-s_{*}\right|+C_{*} F(J),
\end{aligned}
$$

where $K, K^{\prime}, K^{\prime \prime}, K_{j}^{*}, K_{j}^{* *}$, and $C_{*}$ are all positive constants with

$$
K_{0}^{*}>\left|K_{b 0}\right|, \quad\left|K_{b 5}\right|<K_{5}^{*}<\frac{1}{\left|K_{s_{5}}\right|}
$$

Proposition 8.1. Let $J_{k}<I<J<\tilde{J}_{k+1}$ such that $J$ is an immediate successor of $I$. Suppose that

$$
\begin{aligned}
& \left|s_{I}-s_{*}\right|<\varepsilon, \\
& \left|U_{h, \theta}\right|_{I_{+}}-U_{+} \mid<\varepsilon_{1}, \\
& \left|U_{h, \theta}\right|_{I_{-}}-U_{0}(-\infty) \mid<\varepsilon_{2}
\end{aligned}
$$

for some $\varepsilon, \varepsilon_{1}$, and $\varepsilon_{2}>0$. Then there exist positive constants $K, K^{\prime}, K^{\prime \prime}, K_{j}^{*}, K_{j}^{* *}, C_{*}$, and $\tilde{\varepsilon}$, which are independent of $I, J$, and $k$, such that, if $F_{s}(I)<\tilde{\varepsilon}$, then

$$
F_{s}(J)<F_{s}(I) \text {. }
$$

Furthermore, we have

$$
\begin{aligned}
& \left|s_{J}-s_{*}\right|<\varepsilon, \\
& \left|U_{h, \theta}\right|_{J_{+}}-U_{+} \mid<\varepsilon_{1}, \\
& \left|U_{h, \theta}\right|_{J_{-}}-U_{0}(-\infty) \mid<\varepsilon_{2} .
\end{aligned}
$$

Proof. We consider only the case near the strong 1 -shock, since the other cases can be treated in the same way as in the previous sections.

Let $\Lambda$ be the diamond domain between the mesh curves $I$ and $J$.

Case 1: By Lemma 8.2, we have

$$
\begin{aligned}
& L_{1}\left(J_{+}\right)-L_{1}\left(I_{+}\right)=-\left|\beta_{1}\right|, \\
& L_{j}\left(J_{+}\right)-L_{j}\left(I_{+}\right) \leq\left|K_{s_{j}}\right|\left|\beta_{1}\right|+O(1) \Delta, \quad j=2,3,4, \\
& L_{5}\left(J_{+}\right)-L_{5}\left(I_{+}\right) \leq\left|K_{s_{5}}\right|\left|\beta_{1}\right|+O(1) \Delta, \\
& L\left(J_{-}\right)-L\left(I_{-}\right)=0, \\
& Q\left(J_{-}\right)-Q\left(I_{-}\right)=0 .
\end{aligned}
$$




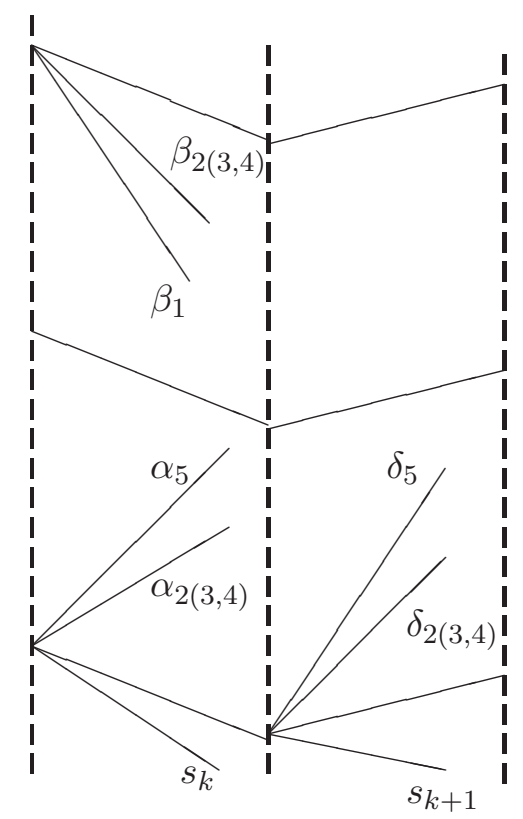

Fig. 15. Case 1

Then we conclude that

$$
L\left(J_{+}\right)-L\left(I_{+}\right) \leq\left(-1+\sum_{j=2}^{5} K_{j}^{*}\left|K_{s_{j}}\right|\right)\left|\beta_{1}\right|+O(1) \Delta,
$$

and

$$
\left.Q\left(J_{+}\right)-Q_{(} I_{+}\right) \leq O(1) \Delta+O(1)\left|\beta_{1}\right| L_{1}\left(J_{+}\right)
$$

Moreover,

$$
\left|s_{k+1}-s_{*}\right| \leq\left|s_{k}-s_{*}\right|+\left|K_{s_{1}}\right|\left|\beta_{1}\right|+O(1) \Delta .
$$

Combining this with the above estimates, and choosing suitable constants $K_{j}^{*}$ and large constants $K, K^{\prime}$, and $K^{\prime \prime}$, we conclude

$$
F_{s}(J) \leq F_{s}(I), \quad \text { for } F_{s}(I) \leq \tilde{\varepsilon} .
$$

Case 2: By Lemma 8.3, we have

$$
\begin{aligned}
& s_{k+1}=s_{k}+O(1)|\boldsymbol{\beta}|, \\
& \delta_{j}=\alpha_{j}+O(1)|\boldsymbol{\beta}|, \quad j=1, \cdots, 5,
\end{aligned}
$$

where $|\boldsymbol{\beta}|=\sum_{j=1}^{5}\left|\beta_{j}\right|$. Then

$$
L\left(J_{-}\right)-L\left(I_{-}\right) \leq-|\boldsymbol{\beta}|
$$

for suitable choice of constants $K_{j}^{* *}$. By choosing sufficiently large $K$, we finally have the desired result.

The proof is complete. 


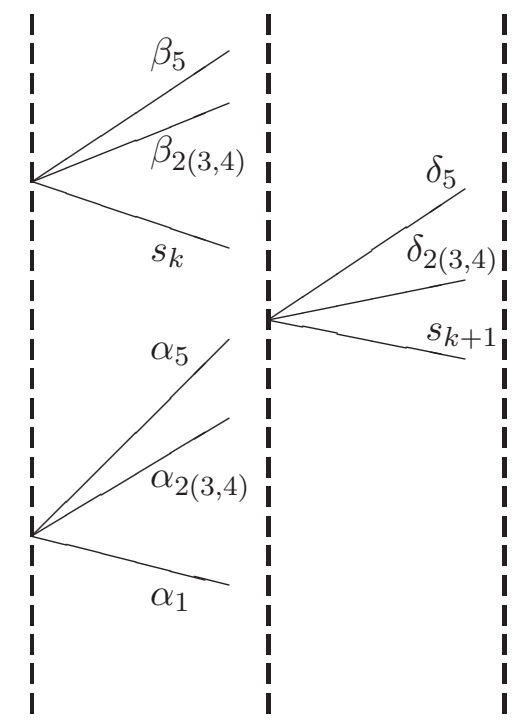

Fig. 16. Case 2

\subsection{Estimates of reaction steps for the strong shock}

By Lemma 4.4, we have

$$
\begin{aligned}
U_{b}-\tilde{U}_{b} & =\left\|Z_{0}\right\|_{\infty} e^{-\Phi_{1} k h} O(h), \\
U_{a}-\tilde{U}_{a} & =\left\|Z_{0}\right\|_{\infty} e^{-\Phi_{1} k h} O(h) .
\end{aligned}
$$

Then

$$
\tilde{s}_{k}-s_{k}=\left\|Z_{0}\right\|_{\infty} e^{-\Phi_{1} k h} O(h) .
$$

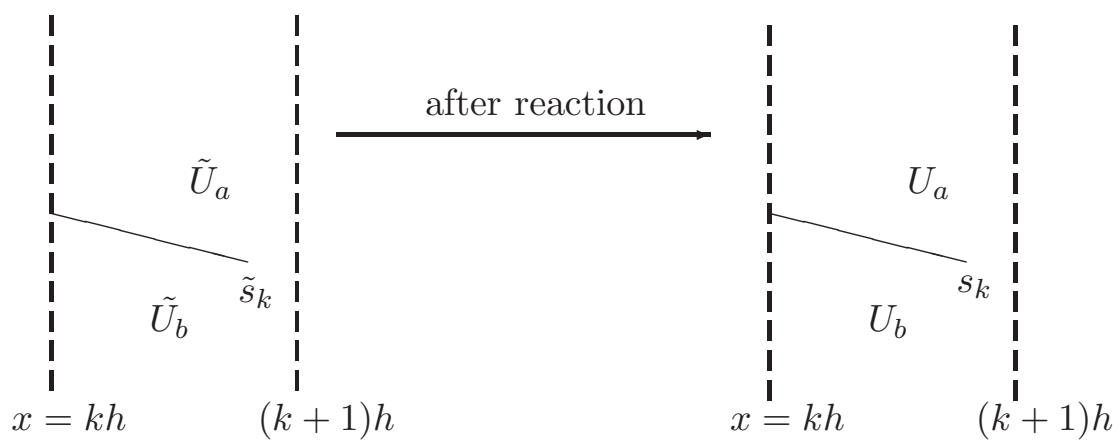

Fig. 17. Change of the strength of the strong shock

As in the previous sections, we still have

$$
F_{s}\left(J_{k}\right)-F_{s}\left(\tilde{J}_{k}\right) \leq C h\left\|w_{5,0}\right\|_{\infty} e^{-\Phi_{1} k h}\left(F_{s}\left(\tilde{J}_{k}\right)+1\right)^{2} .
$$

This gives the uniform bounds on $F_{s}\left(J_{k}\right)$. 


\subsection{Global existence and asymptotic behavior of entropy solutions for the Lipschitz wedge with large angle}

We finally have the following theorem.

Theorem 8.1. Suppose that $0<\arctan \left(\frac{v_{0}(-\infty)}{u_{0}(-\infty)}\right)<\omega_{\text {crit }}$. If $\mathrm{TV}\left(W\left(U_{0}\right)\right)+\mathrm{TV}\left(g^{\prime}\right)$ is sufficiently small, then the fractional-step Glimm scheme can generate a family of approximate solutions $U_{h, \theta}(x, y)$ that have uniformly bounded variation in the $y$-direction. Moreover, there exists a null set $N \subset \Pi_{k=0}^{\infty}(-1,1)$ such that, for every $\theta \in \Pi_{k=0}^{\infty}(-1,1) \backslash N$, there exist a sequence $h_{j} \rightarrow 0$ such that

$$
U_{\theta} \stackrel{L_{\text {loc }}^{1}}{=} \lim _{h_{i} \rightarrow 0} U_{h_{i}, \theta}
$$

is a weak solution to problem (5.1)-(5.2) for system (1.1)-(1.5). Moreover, $U_{\theta}$ has uniformly bounded variation in the $y$-direction.

In the same way as in [2], we have

Theorem 8.2 (Asymptotic behavior). Let $\omega_{\infty}=\lim _{x \rightarrow \infty} \arctan \left(g^{\prime}(x+)\right)$. Then

$$
\lim _{x \rightarrow \infty} \sup _{\chi_{\theta}(x)<y<g(x)}\left|\arctan \left(\frac{v_{\theta}(x, y)}{u_{\theta}(x, y)}-\omega_{\infty}\right)\right|=0,
$$

and

$$
\lim _{x \rightarrow \infty} \sup _{y<\chi_{\theta}(x)}\left|\arctan \left(\frac{v_{\theta}(x, y)}{u_{\theta}(x, y)}\right)\right|=0 .
$$

Acknowledgements: The research of Gui-Qiang Chen was supported in part by the UK EPSRC Science and Innovation Award to the Oxford Centre for Nonlinear PDE (EP/E035027/1), the NSFC under a joint project Grant 10728101, and the Royal Society-Wolfson Research Merit Award (UK). Changguo Xiao was supported in part by the NSFC under a joint project Grant 10728101. Yongqian Zhang was supported in part by NSFC Project 11031001, NSFC Project 11121101, and the 111 Project B08018 (China). 


\section{References}

\section{References}

[1] G.-Q. Chen And D. Wagner, Global entropy solutions to exothermically reacting, compressible Euler equations, J. Differential Equations, 191 (2003), 277-322.

[2] G.-Q. Chen, Y. Q. Zhang, And D. W. Zhu, Existence and stability of supersonic Euler flows past Lipschitz wedges, Arch. Rational Mech. Anal. 181 (2006), 261-310.

[3] G.-Q. Chen, Y. Q. Zhang, And D. W. Zhu, Stabilily of compressible vortex sheets in steady supersonic Euler flows over Lipschitz walls, SIAM J. Math. Anal. 38 (2007), 1660-1693.

[4] S.-X. Chen, Asymptotic behavior of supersonic flow past a convex combined wedge, Chin. Ann. Math. 19B:3 (1998), 255-264.

[5] R. Courant And K. O. Friedrichs, Supersonic Flow and Shock Waves, WileyInterscience, New York, 1948.

[6] C. Dafermos, Hyperbolic Conservation Laws in Continuum Physics, Springer-Verlag: Berlin, 2005.

[7] C. Dafermos and L. Hsiao, Hyperbolic systems of balance laws with inhomegeneity and dissipation, Indiana Univ. Math. J. 31 (1982), 471-491.

[8] J. GLImm, Solutions in the large for nonlinear hyperbolic systems of equations, Comm. Pure Appl. Math. 18 (1965), 697-715.

[9] P. D. Lax, Hyperbolic systems of conservation laws II, Comm. Pure Appl. Math. 10 (1957), $537-566$.

[10] T.-P. LIU, Solutions in the large for the equations of nonisentropic gas dynamics, Indiana Univ. Math. J. 26 (1977), 147-177.

[11] T.-P. LIU, Large-time behaviour of initial and initial-boundary value problems of a general systems of hyperbolic conservation laws, Comm. Math. Phys. 55 (1977), 163-177.

[12] M. Luskin And J. B. Temple, The existence of a global weak solution to the nonlinear waterhammer problem, Comm. Pure Appl. Math. 34 (1982), 697-735.

[13] J. Smoller, Shock Waves and Reaction-Diffusion Equations, Springer-Verlag: New York, 1983.

[14] J. B. Temple, Solutions in the large for the nonlinear hyperbolic conservation laws of gas dynamics, J. Differential Equations, 41 (1981), 96-161.

[15] A. I. Volpert, The space BV and quasilinear equations, Mat. Sb. (N.S), 73 (1967), 255-302 (in Russian); Math. USSR Sb. 2 (1967), 225-267 (in English).

[16] L.-A. YING AND C.-H. WANG, Global solutions of the Cauchy problem for a nonhomogeneous quasilinear hyperbolic system, Comm. Pure Appl. Math. 33 (1980), 579-597.

[17] L.-A. Ying AND C.-H. WANG, Solutions in the large for nonhomogeneous quasilinear hyperbolic systems of equations, J. Math. Anal. Appl. 78 (1980), 440-454.

[18] Y. Q. ZhAng, Global existence of steady supersonic potential flow past a curved wedge with piecewise smooth boundary, SIAM J. Math. Anal. 31 (1999), 166-183. 
[19] Y. Q. Zhang, Steady supersonic flow past an almost straight wedge with large vertex angle, J. Differential Equations, 192 (2003), 1-46. 